NASA Contractor Report 178087

ICASE REPORT NO. $86-19$

NASA-CR-178087

19860017192

$\mathrm{I}$

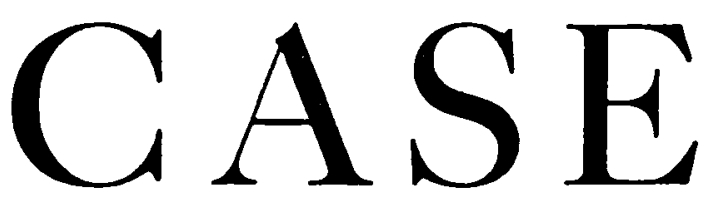

HISTORY MATCHING BY SPLINE APPROXIMATION AND

REGULARIZATION IN SINGLE-PHASE AREAL RESERVOIRS

Tai-yong Lee

Costas Kravaris

John Seinfeld

Contract Nos. NAS $1-17070$, NAS $1-18107$

March 1986

INSTITLTE FOR COMPUTER APPLICATIONS IN SCIENCE AND ENGINEERING

NASA Langley Research Center, Hampton, Virginia 23665

Operated by the Universities Space Research Association

\section{N/SN}

National Aeronautics and

Space Administration

Langley Research Center

Hampton. Virginia 23665

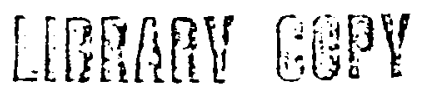

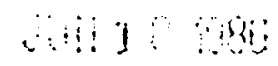

WIIGLEY TISEETRCH CENTER

LIER:ARY, NASA

I.....TOTOU VIREINIA 



\title{
HISTORY MATCHING BY SPLINE APPROXIMATION AND REGULARIZATION IN SINGLE-PHASE AREAL RESERVOIRS
}

\author{
Tai-yong Lee \\ California Institute of Technology \\ Costas Kravaris \\ University of Michigan \\ John H. Seinfeld* \\ California Institute of Technology
}

\begin{abstract}
An automatic history matching algorithm is developed based on bi-cubic spline approximations of permeability and porosity in a single-phase, twodimensional areal reservoir from well pressure data. The regularization feature of the algorithm, the theoretical details of which are described by Kravaris and Seinfeld 3,4 is used to convert the 111 -posed history matching problem into a well-posed problem. The algorithm employs the conjugate gradient method of Nazareth ${ }^{9}$ as its core minimization method. A number of numerical experiments are carried out to evaluate the performance of the algorithm. Comparisons with conventional (non-regularized) automatic history matching algorithms indicate the superiority of the new algorithm with respect to the parameter estimates obtained. A quasioptimal regularization parameter is determined without requiring a priori information on the statistical properties of the observations.
\end{abstract}

\footnotetext{
* The research for the third author was partially supported under the National Aeronautics and Space Administration under NASA Contract Nos. NAS1-17070 and NAS1-18107 while he was in residence at the Institute for Computer Applications in Science and Engineering (ICASE), NASA Langley Research Center, Hampton, VA 23665.
} 



\section{INTRODUCTION}

The process of estimating unknown properties, such as permeability and porosity, in a mathematical reservoir model to give the best fit to measured well pressure and production data is commonly called "history matching." Because the properties in an inhomogeneous reservoir vary with location, conceptually an infinite number of parameters is required for a full description of the reservoir. From a computational point of view, a reservoir simulator contains only a finite number of parameters, corresponding to each grid block or element in the spatial domain. In field scale simulations, it is not unusual for the reservoir domain to consist of the order of 10,000 grid blocks, and consequently 20,000 or more parameters may need to be estimated simultaneously. This potential large dimensionality of the unknown parameters distinguishes the reservoir history matching problem from other parameter estimation problems in science and engineering. Moreover, the standard reservoir history matching problem is mathematically ill-posed, and this inherent ill-posedness, coupled with such a large number of unknown parameters, lies at the root of the difficulties in its solution. The illposedness of the history matching problem manifests itself by numerical instabilities in the estimated parameters. Such instabilities are well documented in the petroleum engineering and hydrology literature ${ }^{1,2}$.

The principal approach that has been used to alleviate ill-conditioning in the parameter estimates is to decrease the number of unknown parameters, and, if possible, utilize any available information to constrain the space of unknown parameters. One commonly used approach for reducing the number of unknown parameters is to divide the reservoir into a relatively small number of zones and to assume uniform properties within each zone. While this 
approach is effective in reducing the number of unknowns, sufficient a priori information is usually not available to enable specification of the zones on any physical basis. Moreover, even though limiting the dimension of the parameter space, zonation does not alleviate the fundamental ill-posed nature of the problem. An alternative to zonation is to use prior information expressed in the form of an assumed probability distribution for the reservoir properties. If certain a priori knowledge is assumed about the parameter mean values and correlations, the history matching objective function can be modified to include a term penalizing the weighted deviations of the parameters from their assumed mean values'. A form of Bayesian estimation can then be used to determine the unknown parameters. While it has been shown that better conditioned estimates may be obtained when a priori statistical information is employed than when it is not sufficient knowledge of the nature of the unknown parameters is generally not available to specify the parameters needed to carry out a Bayesian estimation.

The critical problems in generating an effective algorithm for history matching are twofold: (1) The original problem must be defined in a manner that alleviates the ill-posed nature of the problem; and (2) An efficient computational algorithm must be developed for solving the large, constrained, nonlinear minimization problem that results from any history matching problem.

With respect to the inherent ill-posedness of the history matching problem, Kravaris and Seinfeld $d^{3}, 4$ have shown that the concept of regularization can be applied rigorously to the estimation of spatially-varying parameters in partial differential equations of parabolic type. The regularization-idea, first advanced by Tikhonov ${ }^{18}$, has been widely used in the solution of ill-posed integral equations, but had not heretofore been developed for the estimation 
of parameters in partial differential equations. In short, regularization of a problem refers to solving a related problem, called the regularized problem, whose solution is more "regular" (in a certain sense) than the solution of the original problem and approximates (in a certain sense) the solution of the original problem. More precisely, regularization of an ill-posed problem refers to solving a well-posed problem, whose solution gives a physically meaningful answer to the original ill-posed problem. The regularization formulation of parameter estimation measures the "non-smoothness" of the estimated parameter as a norm of the parameter in an appropriate Hilbert space. No prior information about the parameter is required other than a general idea of the degree of smoothness desired in the estimated field. The only unspecified parameter is that reflecting the relative weight given to the smoothness norm versus the usual least-square objective function.

Banks and co-workers ${ }^{5-8}$ have found that the use of spline representations for spatially-varying parameters in one-dimensional partial differential equations of both parabolic and hyperbolic type leads to well-conditioned estimates. Although their numerical results were obtained for low levels of spline discretization, it seems that the spline representation itself may impart a degree of smoothness to the parameter distribution that could circumvent some of the ill-conditioning inherent in the finite-difference or zonation representation of the unknown parameters. The use of twodimensional, bi-cubic spline approximations for reservoir history matching is an additional new feature of the work reported here.

The object of this work is to present an automatic history matching algorithm based on the concept of regularization together with bi-cubic spline approximations for the estimation of permeability and porosity in a single- 
phase, two-dimensional areal reservoir simulation. The two critical problems cited above are addressed in the algorithm. First, the regularization formulation converts the history matching problem to a mathematically wellposed problem. Second, we use a particularly efficient numerical minimization method, the conjugate gradient method of Nazareth', as the core technique for the minimization. We present the results of extensive numerical testing of the algorithm in which both permeability and porosity distributions are estimated. of particular interest will be the effects of the degree of regularization and of the order of the spline approximation on the behavior of the estimates. 
HISTORY MATCHING BY REGULARIZATION

The problem of history matching may be viewed in a general way by expressing the reservoir model, or simulator, as the nonlinear operator equation,

$$
\mathrm{K} \alpha=\mathrm{u}
$$

where $\alpha$ represents the reservoir parameters, $K$ is the operator representing the reservoir model, and $u$ is the observed portion of the model's output, such as the well pressures. The history matching problem is just the inverse problem to Eq. (1), that is given $u$ and $K$ find $\alpha$. This inverse problem is well-posed if:

(a) For every $u$ there exists a solution $\alpha$

(b) The solution is unique

(c) The solution is stable, i.e., small perturbations in $u$ imply small perturbations in $\alpha$.

If any of (a), (b) or (c) is false, the inverse problem is ill-posed. Establishing uniqueness of $\alpha$ given $u$ for operators $K$ typical of reservoir simulators is an extremely difficult problem, and at this time uniqueness results are only available for very special cases ${ }^{10}$. It can be shown readily, however, that the inverse problem to Eq. (1) for spatially-varying parameters in parabolic partial differential equations is unstable ${ }^{3}$, and the estimation of $\alpha$ from $u$ is an ill-posed problem. As we noted in the Introduction, the illposedness manifests itself by highly ill-conditioned estimates in conventional automatic history matching approaches.

Let us now be more specific and consider unsteady flow of a slightly compressible oil with viscosity $\mu$ in a two dimensional, areal reservoir of unit thickness, spatial domain $\Omega$ and boundary $\partial \Omega$ in which fluid is being withdrawn 
from $\mathrm{N}_{\mathrm{W}}$ wells located at $\left(\mathrm{x}_{\mathrm{W}}, \mathrm{y}_{\mathrm{W}}\right), \mathrm{W}=1,2, \ldots, \mathrm{N}_{\mathrm{W}}$. The fluid properties, $\mu$ and $c$, are assumed to be known whereas the porosity $\phi$ and permeability $k$ are assumed to be unknown. The pressure distribution in the reservoir is governed by

$$
\begin{array}{cc}
c \phi \frac{\partial p}{\partial t}=\nabla \cdot\left(\frac{k}{\mu} \nabla p\right)+\sum_{W=1}^{N_{W}} q_{W} \delta\left(x-x_{W}\right) \delta\left(y-y_{W}\right) \\
\text { in } \Omega \times[0, T] \\
\frac{\partial p}{\partial n}=0 \quad \text { on } \partial \Omega \times[0, T] \\
p(x, y, 0)=p_{0}(x, y) \quad \text { in } \Omega
\end{array}
$$

where $\partial \mathrm{p} / \partial \mathrm{n}$ is the outward normal derivative of $\mathrm{p}$ on the boundary $\partial \Omega$ and $[0, T]$ is the time interval over which data are available. Because of the small size of the well bores relative to the reservoir dimensions, the well flow rates are represented as point sink terms in the pressure equation. If there exist observed pressures at $N_{t}$ times at Nobs locations, $p_{k, n}$ obs, $k=1,2, \ldots, N_{o b s}, n$ $=1,2, \ldots, N_{t}$, then the customary history matching least-squares objective function is

$$
J_{L S}=\sum_{n=1}^{N_{t}} \sum_{k=1}^{N_{O b s}}\left(p_{k, n} \text { obs }-p\left(x_{k}, y_{k}, t_{n}\right)\right)^{2}
$$

The conventional history matching problem can be viewed therefore as a nonlinear optimization problem of minimizing the sum of squares of differences between the observed and predicted pressures subject to the constraint of the reservoir model, Eqs. (2)-(4). 
In the regularization approach we minimize an augmented objective function, called the smoothing functional, denoted by $J_{S M}$, that consists of the sum of the least-squares term, $\mathrm{J}_{\mathrm{LS}}$, and a stabilizing functional, $\mathrm{J}_{S T}$. The stabilizing functional for a parameter $\alpha(\alpha=\phi$ or $k)$ is of the form

$$
J_{S T}=|| \alpha||^{2} H^{3}(\Omega)
$$

where \|\|$^{2} H^{3}(\Omega)$ is a norm defined in the Sobolev space $H^{3}(\Omega)$.* Thus the overall objective function to be minimized is

$$
J_{S M}=J_{L S}+\beta_{\alpha} J_{S T}
$$

Where $\beta_{\alpha}$ is a weighting coefficient chosen to reflect the degree of importance given to JST.

The minimization of $J_{S M}$ is performed over an appropriate finitedimensional subspace of $\mathrm{H}^{3}(\Omega)$, the so-called space of approximants which can be spanned by cubic spline functions. Thus the infinite-dimensional parameter spaces for $k$ and $\phi$ are reduced to finite-dimensional spaces by cubic spline approximations, and the finite dimensional minimization of $J_{S M}$ is carried out by an appropriate numerical minimization method.

\footnotetext{
*The Sobolev space $\mathrm{H}^{3}(\Omega)$ is the set of functions that are square-integrable over $\Omega$ and have square-integrable derivatives up to order 3 . The norm of $\mathrm{H}^{3}(\Omega)$ is given by equation (A.12) of Appendix A.
} 
BICUBIC SPLINE APPROXIMATION OF PERMEABILITY AND POROSITY

A general approach to representing the spatial variation of reservoir properties is through the use of bicubic spline functions, in which a parameter $\alpha(\mathrm{x}, \mathrm{y})$ is represented as

$$
\alpha(x, y)=\sum_{\ell_{y}=1}^{N_{y s}} \sum_{\ell_{x}=1}^{N_{x S}} b_{x}\left(\ell_{x}, x\right) w^{\alpha} \ell_{x}, \ell_{y} b_{y}\left(\ell_{y}, y\right)
$$

where

$$
\begin{aligned}
& b_{x}\left(l_{x}, x\right)=x^{* 4}\left(4-l_{x}+\frac{x}{\Delta x_{S}}\right) \cdot \ell_{x}=1,2, \ldots, N_{x S} \\
& b_{y}\left(l_{y}, y\right)=x^{* 4}\left(4-l_{y}+\frac{y}{\Delta y_{S}}\right) \quad l_{y}=1,2, \ldots, N_{y s}
\end{aligned}
$$

and where $x^{* 4}()$ is the cubic B-spline function, 


$$
\begin{array}{ll}
\frac{x^{3}}{6} & x \in[0,1] \\
\frac{1}{6}+\frac{x-1}{2}+\frac{(x-1)^{2}}{2}-\frac{(x-1)^{3}}{2} & x \in[1,2] \\
x^{* 4}(x)=\frac{4}{6}-(x-2)^{2}+\frac{(x-2)^{3}}{2} & x \in[2,3] \\
\frac{1}{6}-\frac{x-3}{2}+\frac{(x-3)^{2}}{2}-\frac{(x-3)^{3}}{6} & x \in[3,4] \\
0 & \text { otherwise }
\end{array}
$$

$\Delta \mathrm{x}_{\mathrm{S}}$ and $\Delta \mathrm{y}_{\mathrm{S}}$ are the grid distances of the spline grid along the $\mathrm{x}$ - and $\mathrm{y}-$ directions, respectively. With this approximation, $\alpha(x, y)$ is replaced by the set of unknown coefficients, $w^{\alpha} \ell_{x}, l_{y}, \ell_{x}=1,2, \ldots, N_{x s}$ and $\ell_{y}=1,2, \ldots, N_{y s}$. The grid used for spline representation of the unknown properties need not necessarily coincide with that on which the actual reservoir model is solved. The reservoir model will be solved numerically using finite-difference approximations on the block-centered grid system shown in Figure 1. Figure 1 also shows the spline grid system. The finite-difference grid can be expressed compactly as $\mathrm{N}_{\mathrm{X}}=\left\{\mathrm{i}_{\mathrm{x}} \mid \mathrm{i}_{\mathrm{x}}=1,2, \ldots, \mathrm{N}_{\mathrm{x}}\right\}, \mathrm{N}_{\mathrm{y}}=\left\{\mathrm{i}_{\mathrm{y}} \mid \mathrm{i}_{\mathrm{y}}=1,2, \ldots, \mathrm{N}_{\mathrm{y}}\right\}$ and $\mathrm{N}_{\mathrm{N}}=\{\mathrm{i} \mid \mathrm{i}$ $\left.=i_{X}+N_{X}\left(i_{y}-1\right), i_{x} \varepsilon N_{X}, i_{y} \varepsilon N_{y}\right\}=\{i \mid i=1,2, \ldots, N\}$, where $N=N_{X} N_{y} \cdot$ The finite-difference approximation of the pressure equation can be written in compact notation as 
$Q c \phi_{i}\left(p_{i} n-p_{i}^{n-1}\right)=\sum_{j \varepsilon J_{i}} Q_{L}, j \frac{k(i, j)}{\mu}\left(p_{j}^{n}-p_{i}^{n}\right)+\sum_{W=1}^{N_{W}} q_{W} \delta_{i, i_{W}}$

$$
\text { for } i \varepsilon N_{N}, n=1,2, \ldots, N_{t}
$$

where

$$
\begin{aligned}
& J_{i}=\left\{j \mid j=i-N_{X}, i-1, i+1, i+N_{X}\right\} \cap N_{N} \\
& Q=\Delta x \Delta y / \Delta t \\
& Q_{L, j}=\left\{\begin{array}{l}
\Delta y / \Delta x \text { if } j=i-1, i+1 \text { and } j \in N_{N} \\
\Delta x / \Delta y \text { if } j=i-N_{X}, i+N_{X} \text { and } j \in N_{N}
\end{array}\right.
\end{aligned}
$$

$\delta_{i, i_{w}}$ is the Kronecker delta, and $k(i, j)=\left(k_{i}+k_{j}\right) / 2$. The initial condition is $\mathrm{p}_{\mathrm{i}}{ }^{\circ}=\mathrm{p}_{\mathrm{O}}, \mathrm{i} \varepsilon \mathrm{N}_{\mathrm{N}}$.

The least-squares objective function is then written as

$$
J_{L S}=\sum_{i=1}^{N} \sum_{n=1}^{N_{t}} \sum_{k=1}^{N_{\text {Obs }}}\left(p_{k, n} \text { obs }-p_{i}^{n}\right) \delta_{i, i_{k}}
$$

where we assume that $t_{n}-t_{n-1}=\Delta t$ for $n=1, \ldots, N_{t}$. 


\section{HISTORY MATCHING ALCORITHM}

The problem we now seek to solve is to minimize the augmented objective function $J_{S M}$, given by Eq. (7), with respect to the spline coefficients $W^{\alpha} \ell_{X}, \ell_{x}, \ell_{x}=1,2, \ldots, N_{X S}$ and $\ell_{y}=1,2, \ldots, N_{y s}$, subject to the pressure equation (12). To obtain an algorithm to solve this problem two steps are required. First, we must compute the gradient of $J_{S M}$ with respect to each $W^{\alpha} \ell_{X}, \ell_{y}$, and, second, that gradient is then used in a numerical minimization method to minimize $\mathrm{J}_{S M}$. The calculation of these gradients represents the most time consuming part of updating the parameter iterates. In a problem as large as history matching in a candidate algorithm these derivatives must be able to be calculated directly, not requiring the individual derivatives $\partial p_{i}{ }^{n / \partial W^{\alpha}} \ell_{x, \ell_{y}} \cdot{ }^{11,15}$ Those algorithms based on an optimal control, or variational, formulation possess this necessary property. First, we solve the reservoir simulator equation, Eq. (13), from $t=0$ to $t=T$, then solve the adjoint system equation, Eq. (A.7), backward starting from $t=T$ with the terminal condition, Eq. (A.8), to $t=0$, and at the end of each time step during the solution of adjoint system, compute the functional derivative of $J_{L S}$ with respect to permeability, Eq. (A.9), or porosity, Eq. (A.10), at the simulator grid cells. Then, one computes the derivative of $J_{L S}$ with respect to the spline coefficient $W^{\alpha}$, Eq. (A.11), the derivative of $J_{S T}$ with respect to $W^{\alpha}$, Eqs. (A.12-19), and the derivative of $J_{S M}$ with respect to $w^{\alpha}$, Eq. (A.20).

Because of the large dimensionality of $w^{\alpha} \ell_{x, \ell_{y}}$, one seeks to use an algorithm that is as efficient as possible. The essential consideration in the choice of a method is the computational time needed to minimize the objective function. Most of multivariate minimization methods can be divided into two groups: conjugate gradient methods and quasi-Newton methods. The quasi- 
Newton methods are preferred for moderate sized problems, but the conjugate gradient methods become superior to the quasi-Newton methods as the number of variables gets large (Scales 17 suggested 250 as a turning point). Although we treat 30-204 variables in our examples, the number is larger for field applications. The conjugate gradient algorithm of Nazareth ${ }^{9}$ was chosen as the core minimization method in the present code.

The remainder of this work is devoted to the numerical evaluation of the algorithm on the estimation of permeability and porosity distributions in a single-phase, two-dimensional areal reservoir, as modeled by Eq. (2). We want to evaluate the algorithm on a well-defined test problem for which the "true" property distributions are known a priori. For this reason, we will specify the true parameter values, generate our own pressure data by solving the reservoir model with these values, and then try to recover the true parameter values by using the history matching algorithm.

Permeability level and distribution is the principal reservoir property used to match pressure behavior. Porosity is usually better known than permeability, and values from $l o g$ and core data are often used as initial guesses for $\phi$. (Porosity in the aquifer is generally less well known than in the reservoir itself and can be more readily varied than $\phi$ in the reservoir.) Aside from aquifer permeability and porosity which are generally not well known, reservoir permeability is usually more uncertain than porosity.

It is difficult to determine the optimal value of the regularization parameter even if we know the statistical properties of measurement error of the well pressure data. We will choose a set of values of the regularization parameter so that they form a geometric sequence and determine the optimal regularization parameter from the "quasioptimal" value of the regularization 
parameter ${ }^{18}$ which minimizes ||$\beta_{\alpha} \partial w^{\alpha / \partial \beta_{\alpha}} \|_{2}$.

The data for the cases we will study are given in Table 1. Although this set of data is hypothetical, every effort has been made to have the example conform to an actual field simulation.

An important question concerns starting the algorithm. Convergence difficulties are sometimes experienced when the initial guesses of the parameters are far from their actual values. To attempt to alleviate this problem and to generate an algorithm that is as "hands-off" as possible, we begin the estimation by determining the unknown parameters as uniform over the entire region. Thus, to start, we estimate single values of $k$ and $\phi$ for the entire region, called $\bar{k}$ and $\phi$, that minimize $J_{L S}$. These values then serve as starting points for the full history matching algorithm. The rationale behind this strategy is that convergence problems should not be encountered in estimating a single parameter. The single value, while not accurate in its spatial detail, nevertheless serves as a good starting point for the full algorithm. This strategy has been employed in the results to be presented shortly. The single variable minimization is carrried out in our code by the secant method.

Table 2 gives the results of this first step for the estimation of $\phi$ when $k$ is known and the estimation of $k$ when $\phi$ is known. Listed in Table 2 are the true values of $\phi$ and $k$, the initial guesses to start the second method, the minimizing parameter value $\alpha(\alpha=\phi$ or $k)$, and the values of $J_{L S}, J_{S T}$, and $J_{S M}$ for various values of the regularization parameter $\beta$ at the minimum. The true $\phi$ and $k$ surfaces are shown in Figures 2 and 3, respectively. To simulate measurement error, uniformly distributed random numbers are added to the pressure data generated from our presumed true permeability and porosity 
distributions. The resulting data are shown in Figure 4. 


\section{ESTIMATION OF SPATIALLY-VARYING PERMEABILITY AND POROSITY}

We will investigate the effect of the choice of regularization parameter $\left(\beta_{\alpha}\right)$, degree of spline approximation $\left(N_{X s} \times N_{y s}\right)$, and the number of observation wells in the estimation of $\phi$ when $k$ is known and the estimation of $k$ when $\phi$ is known.

We will use six observation wells for values of the regularization parameter $\beta_{\phi}=0,0.01,0.1,1$, and $10 \mathrm{~atm}^{2}$ for the estimation of $\phi$ and $\beta_{k}=0$, $0.01,0.1,1$, and $10 \mathrm{~atm}^{2} /$ darcies $^{2}$ for the estimation of $\mathrm{k}$ and $\mathrm{spline}$ grids $\mathrm{N}_{\mathrm{XS}} \times \mathrm{N}_{\mathrm{ys}}=5 \times 6,7 \times 9$, and $12 \times 17$, where $12 \times 17$ is the maximum possible value for the pressure grid we are using for both the estimation of $\phi$ and the estimation of $k$. As a special case, we will use 18 observation wells for the estimation of $\mathrm{k}$ with $B_{\mathrm{k}}=1 \mathrm{~atm} /$ darcies $^{2}$ adn $\mathrm{N}_{\mathrm{XS}} \times \mathrm{N}_{\mathrm{ys}}=7 \times 9$. Finally, in all our simulations, we assumed that the root mean square error in pressure measurements to be $\sigma=0.3 \mathrm{~atm}$ (thus $\sigma / p_{0}=0.2 \%$ and the corresponding $\mathrm{J}_{\mathrm{LS}}$ value is $18.96 \mathrm{~atm}^{2}$ ).

The estimation of $\phi$ started with uniform value of $\phi=0.184$ and the smoothing functional $\mathrm{J}_{S M}$ was minimized until the change in spline coefficient $W^{\phi}$, and the gradient of $J_{S M}$ with respect to $W^{\phi}$ satisfy the convergence criteria given by

$$
\begin{gathered}
|| W^{\phi, \text { new }}-w^{\phi, o l d}||<\varepsilon_{1} \\
|| \mathrm{G}_{S M}{ }^{\phi}||<\varepsilon_{2}
\end{gathered}
$$

The same strategy was employed for the estimation of $k$, where the starting value of $k=0.241$ darcies ( 0.243 darcies for 18 observation wells) was used. Tables 3 and 4 summarize the history matching results for all the cases studied. 
Effect of Regularization

We expect that, as the regularization parameter increases, the value of the least-squares objective function, $J_{L S}$ at convergence will increase, but the stabilizing functional, JST, will decrease, since a larger value of regularization parameter means more regularization on the parameter to be estimated at the expense of less exact fitting of the observed well pressure data.

This expectation turned out to be true, with some exceptions for the terms $J_{S T}(m+1), m=0$ and 1 , in the stabilizing functional during the estimation of $k$ as shown in Table $4(a)$. That is, $\mathrm{J}_{\mathrm{ST}}(1)$ for $\beta_{\mathrm{K}}=0.1$ $\mathrm{atm}^{2} /$ darcies $^{2}$ is slightly greater than that for $\beta_{k}=0.01 \mathrm{~atm}^{2} /$ darcies ${ }^{2}$ and $\mathrm{J}_{S T}{ }^{(2)}$ for $\beta_{K}=10 \mathrm{~atm}^{2} /$ darcies $^{2}$ is greater than those for $\beta_{k}=0.1$ and 1 $\mathrm{atm}^{2} /$ darcies $^{2}$. But, the total stabilizing functional, $\mathrm{J}_{\mathrm{ST}}$, and its component, $\mathrm{J}_{\mathrm{ST}}{ }^{(4)}$, that represents the third order derivative term and is most important among the four terms, $J_{S T}(m+1), m=0,1,2$, and 3 , decreases strictly without exception as $\beta_{k}$ increases.

Table 3(a) shows that $\mathrm{J}_{S T}{ }^{(4)}$ terms for true $\phi$ is close to that for estimated $\phi$ with $\beta_{\phi}=1 \mathrm{~atm}^{2}$ for which $J_{L S}$ and $\beta_{\phi} J_{S T}$ are balanced, but Table $4(a)$ shows that $J_{S T}{ }^{(4)}$ terms for true $k$ is close to that for estimated $k$ with $\beta_{\phi}$ is betweeen 0.1 and $0.01 \mathrm{~atm}^{2} /$ darcies $^{2}$ for which $J_{L S}$ is $10-100$ times as large as $\beta_{\mathrm{K}} \mathrm{J}_{\mathrm{ST}}$. This means $\mathrm{k}$ can be regularized more easily than $\phi$ can. It is interesting to compare these numerical indicators of performance with the surfaces and profiles in Figures 5 and 6 . The estimated parameter surfaces are too bumpy compared to the true surfaces (Figures 2 and 3 ) when small regularization parameters are used and in the non-regularized case. Note the "bump" in Figure 5.a or the inflection point at $y / y_{L} \approx 0.8$ in curves $1\left(\beta_{K}=0\right)$ and 
$2\left(\beta_{\mathrm{K}}=0.01 \mathrm{~atm}^{2} /\right.$ darcies $\left.^{2}\right)$ of Figure 6 . On the other hand, the parameter estimates become too flat for large values of regularization parameters, as shown in Figure $5 . c$ and curve 5 in Figure 6, as compared to Figure 2 and curve 6 in Figure 6.

To determine the optimal regularization parameter, ||$\beta_{\alpha} d W^{\alpha} / d \beta_{\alpha}||_{2}$ can be approximated by $\left\|\left(W_{2} \alpha_{-} W_{1} \alpha\right) /\left(\ln \beta_{\alpha, 2}-\ln \beta_{\alpha, 1}\right)\right\|_{2}$ where $\beta_{\alpha, 1}$ and $\beta_{\alpha, 2}$ denote two different regularization parameters and $W_{1}^{\alpha}$ and $W_{2}{ }^{\alpha}$, respectively, are the corresponding spline coefficients that minimize $J_{S M}$ and \|\|$_{2}$ denotes Euclidean vector norm. Table 5 summarizes the results for the estimation of $\phi$ and the estimation of $k$. The optimal $\beta_{\phi} \approx 0.1$ to $1 \mathrm{~atm}^{2}$ for the estimation of $\phi$ and the optimal $\beta_{k} \approx 0.01$ to $0.1 \mathrm{~atm}^{2} / \mathrm{darcies}^{2}$ for the estimation of $\mathrm{k}$ which agree with the above investigation.

Table 4 also shows that, in the estimation of $k$, the $\mathrm{JST}_{\mathrm{ST}}(1)$ term, which is proportional to the Euclidean norm of $k(x, y)$, in the domain $\Omega$ is smaller than that calculated from the true $k$. This behavior can be explained from Eq. (2), which shows that the pressure value is governed by the gradient of $k$ with respect to the space variables rather than the value of $k$ itself. Thus the value of $k$ can be reduced to some extent without changing the values of pressure significantly during the estimation of $k$.

Effect of Spline Approximation $\left(\mathrm{N}_{\mathrm{XS}} \mathrm{xN}_{\mathrm{ys}}\right)$

The measurement error ( $J_{L S}$ for true parameters) is $18.96 \mathrm{~atm}^{2}$ while $J_{L S}$ for $\beta_{\phi}=0$ is $20.67 \mathrm{~atm}^{2}$ for the estimation of $\phi$ and $J_{L S}$ for $B_{K}=0$ is 21.26 $a^{2}{ }^{2}$ for the estimation of $k$. This can be explained by the fact that the spline approximation has the effect of smoothing instead of fitting the noisy measured data in detail. 
It is clear that the measured data can be better fit with more parameters, thus we expect that values of $J_{L S}$ should decrease as the dimension of spline grid, $\mathrm{N}_{\mathrm{XS}} \mathrm{XN} \mathrm{N}_{\mathrm{ys}}$, increases. At the same time the estimated parameters are expected to be less regular for larger values of $\mathrm{N}_{\mathrm{XS}} \mathrm{xN}_{\mathrm{ys}}$. This expectation turns out to be true in our examples in the estimation of both $\phi$ and $k$. The value of $\mathrm{J}_{S T}{ }^{(4)}$ for the true $\phi$ is closer to that for the estimated $\phi$ when $\mathrm{N}_{\mathrm{XS}} \mathrm{XN} \mathrm{yS}=7 \times 9$, and the values of $\mathrm{J}_{\mathrm{ST}}{ }^{(4)}$ for the true $k$ lies between those of the estimated $\mathrm{k}$ for $\mathrm{N}_{\mathrm{xS}} \mathrm{xN}_{\mathrm{ys}}=7 \times 9$ and $12 \times 17$. However, if we want the values of $\mathrm{J}_{\mathrm{ST}}(4)$ not to be greater than that of true $k$, we conclude that $\mathrm{N}_{\mathrm{XS}} \mathrm{XN}_{\mathrm{ys}}=7 \times 9$ is the best value for the $10 \times 15$ pressure grid in our example. Figures 7 and 8 show the effect of spline approximation on the estimation of $\phi$ and $k$, respectively. We can observe ill-conditioning in the estimation of $\mathrm{k}$ from Figure $8 \mathrm{c}\left(\mathrm{N}_{\mathrm{XS}} \mathrm{XN}_{\mathrm{ys}}=12 \times 17\right)$ which can be explained since the ratio of spline grid to pressure grid $(h)$ is only 1.09, so that we have 204 unknown spline coefficients to be estimated as compared with 210 measured pressure data.

Effect of Number of Observation Wells

The regularization effect is relatively less important for more observation wells and thus the values of $J_{S T}$ and the terms in $J_{S T}, J_{S T}(m+1), m$ $=0,1,2$, and 3 are larger as shown in Table 4(c). As one can see in Figure 9, the estimated $k$ for 18 observation wells is closer to the true $k$ than that for six observation wells. Note that $J_{L S}$ for 18 observation wells case is closer to the true value than that for six observation wells with any set of $k$ and $\mathrm{N}_{\mathrm{XS}} \mathrm{xN}$ ys is. 
CONVERGENCE OF THE ALGORITHM

When we seek the minimum of $J_{S M}$ with respect to $W^{\alpha}(\alpha=\phi$ or $k)$ with the conjugate gradient algorithm, we need up to $\mathrm{N}_{\mathrm{XS}} \mathrm{xN}$ ys conjugate directions to find the approximation of the inverse of the Hessian matrix. However, the algorithm employed here uses 5 to 10 conjugate directions to find the approximation of the inverse of the Hessian matrix. On the whole, the algorithm requires 10 to 20 different inverse Hessian matrix evaluations which correspond to 0.5 to 1 hour of CPU time on a VAX11-780 for a single history match.

The algorithm determines the minimum of $\mathrm{J}_{S M}\left(\mathrm{~W}^{\alpha}+{\mathrm{S} \delta \mathrm{W}^{\alpha}}^{\alpha}\right)$ along $\mathrm{s}$, the step size, by trial and error. In some cases, if $s$ is too large, some elements of $W^{\alpha}+s \delta W^{\alpha}$ have negative values that are physically impossible. Thus, in the implementation of the algorithm a limit on the size of $s$ was employed. 


\section{CONCLUSIONS}

In this study we have developed and tested an automatic history matching algorithm for estimating spatially-varying porosity and permeability in a single phase areal reservoir. The algorithm is based on spline approximations of the parameters and a regularization formulation. In the regularization approach to parameter estimation by introducing the stabilizing functional as a measure of "non-smoothness," one can control the properties of the parameter estimates as well as the history match.

We have presented results of a detailed numerical evaluation of the performance of the history matching method. In this example, we found that the permeability distribution is estimated somewhat better than the porosity distribution at comparable levels of spline approximation and degree of regularization. It was also found that increasing the value of the regularization parameter leads to estimated property distributions that are smoother than those obtained for smaller values of the regularization parameter. Some exceptions to this behavior were found at small values of the regularization parameter that can be attributed to inherent numerical illconditioning in estimation problems of this size. There appears to be an optimal level of spline approximation in the case of the example studied. The optimum was a $7 \times 9$ grid, for which the ratio of the size of the spline grid to that of the pressure grid is 2.5. This optimal value of approximation appears to represent a trade-off between a low dimensional spline grid that has as few unknown parameters as possible and a high dimensional spline grid that is more able to represent the details of property distributions but introduces more unknowns and therefore inherently more ill-conditioning in the optimization step. 
Based upon the optimal spline approximation, the optimal regularization parameters are $B_{\phi}=0.1$ to $1 \mathrm{~atm}^{2}$ for the estimation of $\phi$ and $\beta_{k}=0.01$ to 0.1 $\operatorname{atm}^{2} /$ darcies $^{2}$ for the estimation of $k$ which are determined from the "quasioptimal" condition of regularization and give the same magnitude of the values of measure of "non-smoothness" compared to the true profiles.

Finally, we can suggest a history matching strategy:

1. Choose simulator and spline grid systems. The number at the spline coefficients need not be as large as the number of simulator grid cells.

2. Find uniform initial guess of parameter to be estimated which minimizes $\mathrm{J}_{\mathrm{LS}}$ and calculate $\mathrm{J}_{\mathrm{LS}} / \mathrm{J}_{\mathrm{ST}}$ at convergence.

3. Choose the regularization parameter value approximately the same as $\mathrm{J}_{\mathrm{LS}} / \mathrm{J}_{\mathrm{ST}}$ above and find a set of spline coefficients that minimizes $\mathrm{J}_{\mathrm{SM}}$.

4. Step (3) can be repeated to evaluate the result for the different regularization parameter values around the $\mathrm{J}_{\mathrm{LS}} / \mathrm{J}_{\mathrm{ST}}$ value determined in Step (2), so that we can find the "optimum" value of regularization parameter discussed in the previous section. 
APPENDIX A. GRADIENT OF THE OBJECTIVE FUNCTION WITH RESPECT TO THE UNKNOWN PARAMETERS

Let us begin by supposing that we want to minimize the least-squares objective function, $J_{L S}$, given by Eq. (5), with respect to permeability $k$ and porosity $\phi$ subject to Eqs. (2)-(4). By adjoining the model of Eq. (2) to $J_{L S}$ by means of an adjoint function $\psi(x, y, t)$,

$$
\begin{aligned}
\bar{J}_{L S} & =\iiint_{\Omega}\left\{\sum_{n=1}^{N_{t}} \sum_{k=1}^{N_{o b s}}\left(p_{k, n}^{o b s}-p(x, y, t)\right)^{2} \delta\left(x-x_{k}\right) \delta\left(y-y_{k}\right) \delta\left(t-t_{n}\right)\right. \\
& +\psi(x, y, t)\left[-c \phi \frac{\partial}{\partial t} p(x, y, t)+\nabla \cdot\left(\frac{k}{\mu} \nabla p(x, y, t)\right)\right. \\
& \left.\left.+\sum_{W=1}^{N_{W}} q_{w} \delta\left(x-x_{w}\right) \delta\left(y-y_{w}\right)\right]\right\} d t d x d y
\end{aligned}
$$

then minimizing $\bar{j}_{L S}$ leads to the following equations governing $\psi(x, y, t)$,

$$
\begin{array}{ll}
c \phi \frac{\partial}{\partial t} \psi(x, y, t)=-\nabla \cdot\left(\frac{k}{\mu} \psi(x, y, t)\right) & \\
-2 \sum_{n=1}^{N_{t}} \sum_{k=1}^{N_{o b s}}\left\{\left(p_{k, n}^{o b s}-p(x, y, t)\right) \delta\left(x-x_{k}\right) \delta\left(y-y_{k}\right) \delta\left(t-t_{n}\right)\right\} & \text { in } \Omega \times[0, T] \\
\frac{\partial \psi}{\partial n}=0 & \text { on } \partial \Omega \times[0, T] \\
\psi(x, y, T)=0 & \text { in } \Omega
\end{array}
$$

where $n$ has the direction outward normal to the boundary, and the functional derivatives of $J_{L S}$ with respect to $k$ and $\phi$ at $(x, y) \in \Omega$ are 


$$
\begin{aligned}
G_{L S}^{k}(x, y) & =-\frac{1}{\mu} \int_{0}^{T} \nabla \psi(x, y, t) \cdot \nabla p(x, y, t) d t \\
G_{L S}^{\phi}(x, y) & =-c \int_{0}^{T} \psi(x, y, t) \frac{\partial}{\partial t} p(x, y, t) d t \\
& =c \psi(x, y, 0) p_{0}(x, y)+c \int_{0}^{T}\left\{\left[\frac{\partial}{\partial t} \psi(x, y, t)\right] p(x, y, t)\right\} d t
\end{aligned}
$$

The first order necessary condition for a local minimum of $J_{L S}$ is that $p$ and $\psi$ satisfy Eqs. (2) $-(4)$ and (A.2)-(A.4), respectively, and that $G_{L S}^{k}(x, y)=0$ (for the estimation of $k$ ) or $G_{L S}^{\phi}(x, y)=0$ (for the estimation of $\phi$ ) for alf $(x, y) \in \Omega$. The gradients $G_{L S}^{k}(x, y)$ and $G_{L S}^{\phi}(x, y)$ are used in the socalled optimal control algorithms for history matching. As noted, since these gradients can be calculated directly without requiring the sensitivity coefficients, $\partial p / \partial k$ and $\partial p / \partial \phi$, these optimal control algorithms are computationally attractive for history matching.

The adjoint equations (A.2)-(A.4) can be written in a finite difference form corresponding to Eq. (12) as

$$
\begin{aligned}
Q \subset \phi_{i}\left(\psi_{i}^{n+1}-\psi_{i}^{n}\right) & =-\sum_{j \in J_{j}} Q_{L, j}\left(\psi_{j}^{n}-\psi_{i}^{n}\right) \\
& -2 \sum_{k=1}^{N \text { obs }}\left(p_{k, n}^{\text {obs }}-p_{j}^{n}\right) \delta_{i, i_{k}} \\
& \text { for } i \in \mathbb{N}_{N} \text { and } n=1,2, \ldots, N_{t}
\end{aligned}
$$

$$
\psi_{i}^{N_{t}+1}=0 \quad i \in \mathbb{N}_{N}
$$


where $J_{i}=\left\{i-N_{x}, i-1, i+1, i+N_{x}\right\} \cap N_{N}$ and the derivatives of $J_{L S}$ with respect to $k_{i}$ and $\phi_{i}$ are

$$
\begin{aligned}
G_{L S, i}^{k} & =-\frac{1}{\mu} \sum_{n=1}^{N_{t}} \sum_{j \in J_{i}} Q_{L, j}\left(\psi_{j}^{n}-\psi_{i}^{n}\right)\left(p_{j}^{n}-p_{i}^{n}\right) \\
G_{L S, i}^{\phi} & =-Q c \sum_{n=1}^{N_{t}} \psi_{i}^{n}\left(p_{i}^{n}-p_{i}^{n-1}\right) \quad \text { for } i \epsilon N_{N} \\
& =Q c\left\{\psi_{i}^{1}+\sum_{n=1}^{N_{t}}\left(\psi_{i}^{n+1}-\psi_{i}^{n}\right) p_{i}^{n}\right\}
\end{aligned}
$$

for $i \in N_{N}$

In our algorithm, $k$ and $\phi$ are represented by the bi-cubic spline approximation Eq. (8), and the actual unknown parameters are the coefficients, $w_{l_{x}, l_{y}}^{k}$ and $w_{l_{x}, l_{y}}^{\phi}$. Thus, we need to obtain the derivatives of the overall objective functional $J_{S M}$ with respect to $w_{l_{x}, l_{y}}^{k}$ and $w_{l_{x}, l_{y}}^{\phi}$. These gradients are then the values used directiy in the conjugate gradient minimization method.

Let the $N_{x s} \times N_{x}$ matrix $B_{x}$ have elements $B_{x, l_{x}, i_{x}}=b_{x}\left(l_{x},\left(i_{x}-\frac{1}{2}\right) \Delta x\right)$ and the $N_{y s} \times N_{y}$ matrix $B_{y}$ have elements $B_{y, l_{y}, i_{y}}=b_{y}\left(l_{y},\left(i_{y}-\frac{1}{2}\right) \Delta y\right)$. Then the derivative of $J_{L S}$ with respect to the elements of $W^{\alpha}(\alpha=k$ or $\phi)$ is

$$
\begin{aligned}
& G_{L S, \ell_{x}, l_{y}}^{W^{\alpha}}=\sum_{i_{x}=1}^{N_{x}} \sum_{i_{y}=1}^{N_{y}} B_{x, \ell_{x}, i_{x}} G_{L S, i}^{\alpha} B_{y, \ell_{y}, i_{y}} \\
& \ell_{x}=1, \ldots, N_{x S} \text { and } \ell_{y}=1, \ldots, N_{y s}
\end{aligned}
$$

where $i=i_{x}+N_{x}\left(i_{y}-1\right)$. Thus Eq. (A.11) relates the gradient of the leastsquares objective function with respect to the spline coefficients to that 
with respect to the individual reservoir parameters at each grid point of the simulator.

Eq. (A.11) expresses the gradient of the least-squares portion, $J_{L S}$, of the overall objective function, $\mathrm{J}_{S M}$. We need to obtain the gradient of $\mathrm{J}_{S M}$. Let us consider the second component of $\mathrm{J}_{S M}$, namely the stabilizing functional, $J_{S T}$, given by Eq. (6) which is

$$
\begin{aligned}
J_{S T}=\zeta_{1} J_{S T}^{(1)}+\zeta_{2} J_{S T}^{(2)}+\zeta_{3} J_{S T}^{(3)}+\zeta_{4} J_{S T}^{(4)} \\
J_{S T}^{(1)}=\int_{0}^{N} \int_{0}^{N} \alpha^{2}(\xi, \eta) d \xi d n \\
J_{S T}^{(2)}=\int_{0}^{N} \int_{0}^{N}\left[\left(\frac{\partial \alpha}{\partial \xi}\right)^{2}+\left(\frac{\partial \alpha}{\partial \eta}\right)^{2}\right] d \xi d n \\
J_{S T}^{(3)}=\int_{0}^{N} \int_{0}^{N}\left[\left(\frac{\partial^{2} \alpha}{\partial \xi^{2}}\right)^{2}+2\left(\frac{\partial^{2} \alpha}{\partial \xi \partial \eta}\right)^{2}+\left(\frac{\partial^{2} \alpha}{\partial n^{2}}\right)^{2}\right] d \xi d n \\
J_{S T}^{(4)}=\int_{0}^{N} \int_{0}^{N}\left[\left(\frac{\partial^{3} \alpha}{\partial \xi^{3}}\right)^{2}+3\left(\frac{\partial^{3} \alpha}{\partial \xi^{2} \partial \eta}\right)^{2}+3\left(\frac{\partial^{3} \alpha}{\partial \xi \partial \eta^{2}}\right)^{2}+\left(\frac{\partial^{3} \alpha}{\partial \eta^{3}}\right)^{2}\right] d \xi d n
\end{aligned}
$$

where $\zeta_{1}>0, \zeta_{2} \geq 0, \zeta_{3} \geq 0, \zeta_{4}>0$ and $\xi=x / \Delta x$ and $n=y / \Delta y$. Using $\xi$ and $n$ rather than $x$ and $y, b_{x}\left(l_{x}, x\right)$ and $b_{y}\left(l_{y}, y\right)$ in Eqs. (9) and (10) and their derivatives are

$$
\begin{aligned}
& b_{x}\left(l_{x}, x\right)=x \star^{4}\left(4-l_{x}+\xi / h_{x}\right) \\
& b_{y}\left(l_{y}, y\right)=x \star^{4}\left(4-l_{y}+n / h_{y}\right) \\
& \frac{d^{m}}{d \xi^{m}} b_{x}\left(l_{x}, x\right)=\frac{1}{h_{x}^{m}} x \star^{4(m)}\left(4-l_{x}+\xi / h_{x}\right), m=1,2,3
\end{aligned}
$$


26

$$
\frac{d^{m}}{d n^{m}} b_{y}\left(l_{y}, y\right)=\frac{1}{h_{y}^{m}} x \star^{4(m)}\left(4-l_{y}+n / h_{y}\right), m=1,2,3
$$

where $x^{*^{4(m)}}(\cdot)$ denotes $m$-th derivatives of $x^{*}(\cdot), h_{x}=\Delta x_{s} / \Delta x$, and $h_{y}=$ $\Delta y_{s} / \Delta y$. Combining Eqs. (8), (A.12), and (A.13)

$$
\begin{aligned}
& H_{S T, l_{x}, l_{y}, m_{x}, m_{y}}=2 \zeta_{1} A_{l_{x}, m_{x}}^{0 x} A_{l_{y}, m_{y}}^{0 y} \\
& +\zeta_{2}\left(A_{l_{x}, m_{x}}^{1 x} A_{l_{y}, m_{y}}^{0 y}+A_{l_{x}, m_{x}}^{0 x} A_{l_{y}, m_{y}}^{1 y}\right) \\
& +\zeta_{3}\left(A_{l_{x}}^{2 x}, m_{x} A_{l_{y}}^{0 y}, m_{y}+2 A_{l_{x}, m_{x}}^{1 x} A_{l_{y}, m_{y}}^{1 y}\right. \\
& \left.+A_{l_{x}}^{0 x}, m_{x} A_{l_{y}, m_{y}}^{2 y}\right) \\
& +\zeta_{4}\left(A_{l_{x}}^{3 x}, m_{x} A_{l_{y}}^{0 y}, m_{y}+3 A_{l_{x}}^{2 x}, m_{x} A_{l_{y}}^{1 y}, m_{y}\right. \\
& \left.+3 A_{l_{x}, m_{x}{ }^{1 x} A_{y}, m_{y}}^{2 y}+A_{l_{x}, m_{x}}^{0 x} A_{l_{y}, m_{y}}^{3 y}\right) \\
& G_{S T, l_{x}, l_{y}}=\sum_{m_{x}=1}^{N_{x S}} \sum_{m_{y}=1}^{N_{y s}} H_{S T, l_{x}, l_{y}, m_{x}, m_{y}} W_{m_{x}, m_{y}}^{\alpha} \\
& J_{S T}=\sum_{l_{x}=1}^{N} \sum_{\ell_{y}=1}^{N} \frac{1}{2} G_{S T, l_{x}, l_{y}}^{W} W_{l_{x}, l_{y}}^{\alpha}
\end{aligned}
$$

where

$$
\begin{gathered}
A_{\ell_{x}, m_{x}}^{(m) x}=h_{x}^{1-2 m} \int_{0}^{N} x^{x s^{-3}}(m)\left(4-\ell_{x}+\frac{\xi}{h_{x}}\right) x *^{4(m)}\left(4-m_{x}+\frac{\xi}{h_{x}}\right) d\left(\frac{\xi}{h_{x}}\right) \\
m=0,1,2,3
\end{gathered}
$$




$$
\begin{gathered}
A_{l_{x}, l_{y}}^{(m) y}=h_{y}^{1-2 m} \int_{0}^{N_{y s}-3} x *^{4(m)}\left(4-l_{y}+\frac{n}{h_{y}}\right) x *^{4(m)}\left(4-m_{y}+\frac{\eta}{h_{y}}\right) d\left(\frac{n}{h_{y}}\right) \\
m=0,1,2,3
\end{gathered}
$$

or in the matrix form,

$$
\begin{aligned}
& A^{0 X}=\frac{h_{x}}{7 !}\left[\begin{array}{rrrrrrrrr}
20 & 129 & 60 & 1 & & & & & \\
129 & 1208 & 1062 & 120 & 1 & & & & \\
60 & 1062 & 2396 & 1191 & 120 & 1 & & \\
1 & 120 & 1191 & 2416 & 1191 & 120 & 1 & & \\
& --- & --- & --- & --- & --- & --- & --. & -- \\
& & 1 & 120 & 1191 & 2416 & 1191 & 120 & 1 \\
& & & 1 & 120 & 1191 & 2396 & 1062 & 60 \\
& & & & 1 & 120 & 1062 & 1208 & 129 \\
& & & & & 1 & 60 & 129 & 20
\end{array}\right] \\
& A^{1 \times}=\frac{1}{5 ! h} \times\left[\begin{array}{rrrrrrrrr}
6 & 7 & -12 & -1 & & & & & \\
7 & 40 & -22 & -24 & -1 & & & & \\
-12 & -22 & 74 & -15 & -24 & -1 & & & \\
-1 & -24 & -15 & 80 & -15 & -24 & -1 & & \\
& --- & --- & --- & --- & -- & --- & --- & \\
& & -1 & -24 & -15 & 80 & -15 & -24 & -1 \\
& & & -1 & -24 & -15 & 74 & -22 & -12 \\
& & & & -1 & -24 & -22 & 40 & 7 \\
& & & & & -1 & -12 & 7 & 6
\end{array}\right]
\end{aligned}
$$




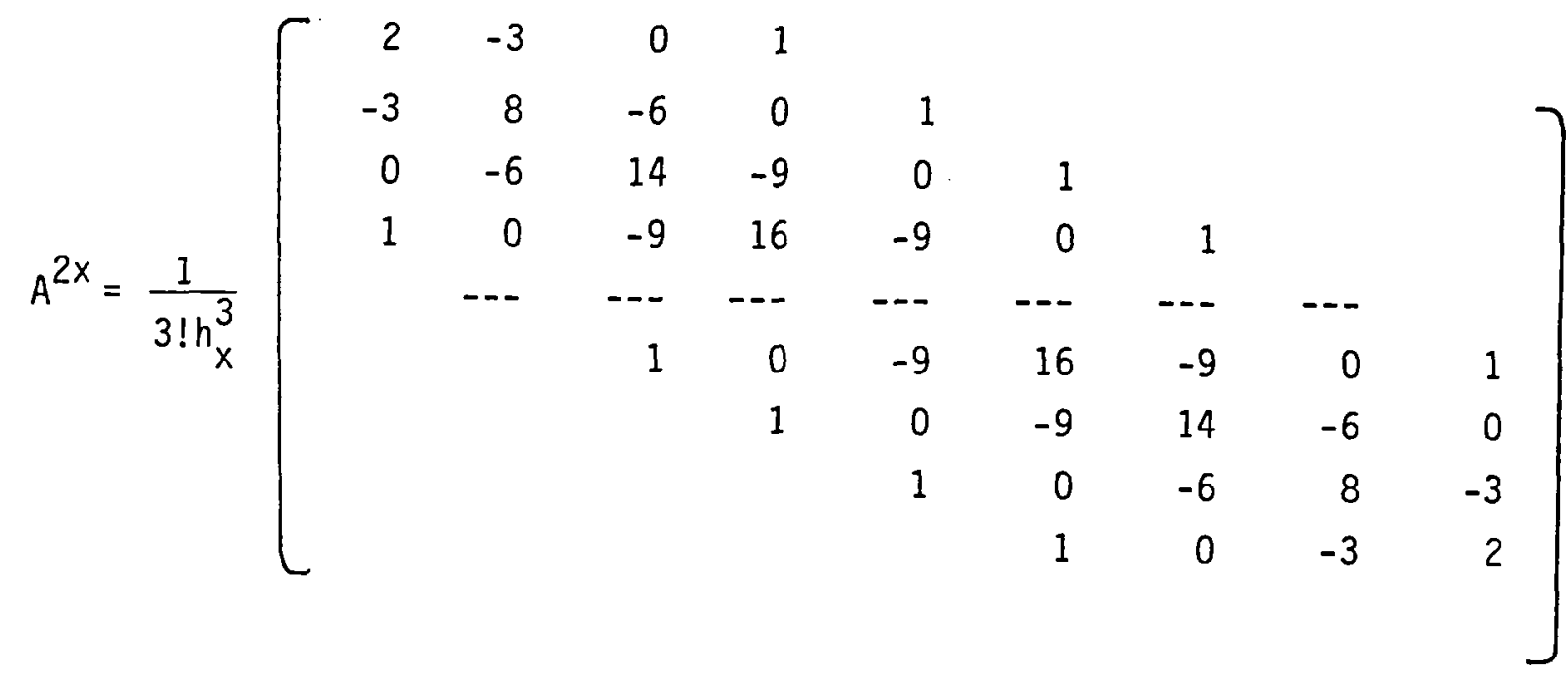

$\left.A^{3 x}=\frac{1}{h_{x}^{5}} \quad \begin{array}{rrrrrrrrr}1 & -3 & 3 & -1 & & & & & \\ -3 & 10 & -12 & 6 & -1 & & & & \\ 3 & -12 & 19 & -15 & 6 & -1 & & & \\ -1 & 6 & -15 & 20 & -15 & 6 & -1 & & \\ & --- & --- & --- & --- & --- & --- & --- & \\ & & -1 & 6 & -15 & 20 & -15 & 6 & -1 \\ & & & -1 & 6 & -15 & 19 & -12 & 3 \\ & & & & -1 & 6 & -12 & 10 & -3 \\ & & & & & -1 & 3 & -3 & 1\end{array}\right]$

(A.19)

and $A^{0 y}, A^{1 y}, A^{2 y}$, and $A^{3 y}$ have the analogous expressions to $E q .(A .19)$.

Finally, we obtain the gradient of overall objective function $J_{S M}$ with respect to $w^{\alpha}$ as

$$
G_{S M, \ell_{x} \ell_{y}}^{W^{\alpha}}=G_{L S, \ell_{x} \ell_{y}}^{W^{\alpha}}+B_{\alpha} G_{S T, \ell_{x} \ell_{y}}^{W^{\alpha}}
$$


Weighting Factor $\zeta_{m+1}, m=0,1,2$, and 3 in Eq. (A.12.1)

In the stabilizing functional in Eq. (A.12.1), we have four undetermined constants, namely the weighting factors, $\zeta_{m+1}, m=0,1,2$, and 3 , which are arbitrary except for the conditions that $\zeta_{1}>0, \zeta_{2} \geqslant 0, \zeta_{3} \geqslant 0$, and $\zeta_{4}>0$. We can set these constants in a systematic way by using the fact that we want the four terms in Eq. (A.12.1), each of which is a weighting factor $\zeta_{m+1}$ multiplied by $J_{S T}^{(m+1)}$, described in Eqs. (A.12.2)-(A.12.5), to be of about equal magnitude. We assume that $h_{x}$, the ratio of the size of the spline grid to that of the simulator grid along $x$-direction is not much different from $h_{y}$, the ratio along the $y$-direction, and we let $h=\left(h_{x} h_{y}\right)^{\frac{1}{2}}$. Then, the ratio of terms $J_{S T}^{(m+1)}, m=0,1,2$, and 3 in Eq. (A.12) is approximately

$$
J_{S T}^{(1)}: J_{S T}^{(2)}: J_{S T}^{(3)}: J_{S T}^{(4)}=h^{2}: 1: \frac{1}{h^{2}}: \frac{1}{h^{4}}
$$

and this suggests values for the weighting factors, $\zeta_{m+1}, m=0,1,2$, and 3 as $\zeta_{1}=1, \zeta_{2}=h^{2} ; \zeta_{3}=h^{4} ; \zeta_{4}=h^{6}$, where $\zeta_{1}$ is independent of $h$. 
NOTATION

$A_{x}^{(m)}$

$A_{y}^{(m)}$

$A^{(m) x}$

${ }^{\prime} m_{x}$

$A_{l}^{(m) y}$

$b_{x}\left(e_{x}, x\right)$

$b_{y}\left(e_{y}, y\right)$

$B_{x}$

$B_{y}$

c

matrix defined by Eq. (A.19), $m=0,1,2$, and 3

matrix defined by Eq. (A.19), $m=0,1,2$, and 3

quantity defined by Eq. (A.17)

quantity defined by Eq. (A.18)

cubic spline function defined by Eq. (9)

cubic spline function defined by Eq. (10)

$N_{x s} x N_{x}$ matrix of spline function values

$\mathrm{N}_{\mathrm{ys}} \mathrm{xN}$ matrix of spline function values

compressibility, $\mathrm{atm}^{-1}\left[\mathrm{~Pa}^{-1}\right]$

$G_{L S}^{k}(x, y), G_{L S}^{\phi}(x, y)$ functional derivative of $J_{L S}$ with respect to $k$ and $\phi$ at $(x, y)$

$G_{L S, i}^{k}, G_{L S, i}^{\phi} \quad$ derivative of $J_{L S}$ with respect to the values of $k$ and $\phi$ at the grid point of the simulator, $i=1,2, \ldots, N$

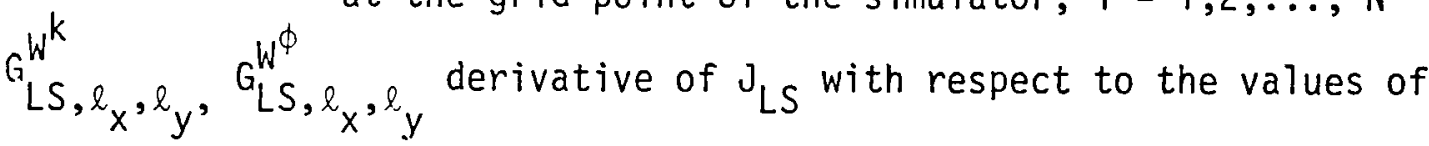

$$
W_{l_{x}, l_{y}}^{k} \text { and } w_{l_{x}, l_{y}}^{\phi}
$$

$G_{S M, l_{x}, l_{y},}^{W^{k}} G_{S M, l_{x}, l_{y}}^{W^{\phi}}$ derivative of $J_{S M}$ with respect to the values of

$$
W_{l_{x}, l_{y}}^{k} \text { and } W_{l_{x}, l_{y}}^{\phi}
$$

$G_{S T, l_{x}, l_{y}}^{W^{k}}, G_{S T, l_{x}, l_{y}}^{W^{\phi}}$ derivative of $J_{S T}$ with respect to the values of

$$
W_{l_{x}, l_{y}}^{k} \text { and } W_{l_{x}, l_{y}}^{\phi}
$$




$$
\begin{aligned}
& H^{3}(\Omega) \quad \text { Sobolev space of order } 3 \text { on the domain } \Omega \\
& H_{S T}, l_{x}, l_{y}, m_{x}, m_{y} \text { quantity defined by Eq. (A, 14) } \\
& \text { h } \quad\left(h_{x} h_{y}\right)^{\frac{1}{2}} \\
& h_{x} \quad \Delta x_{s} / \Delta x \\
& h_{y} \quad \Delta y_{s} / \Delta y \\
& i, i_{x}, i_{y} \quad \text { indices for simulator grid, } i=i_{x}+N_{x}\left(i_{y}-1\right) \\
& \text { JLS least squares objective function, Eq. (5) } \\
& \mathrm{J}_{S M} \quad \text { smoothing functional, Eq. (7) } \\
& \mathrm{J}_{\mathrm{ST}} \quad \text { stabilizing functional, Eq. (6) } \\
& \mathrm{J}_{\mathrm{ST}}^{(\mathrm{m}+1)} \quad \text { terms in stabilizing functional defined by Eq. (A.12), } \\
& m=0,1,2 \text {, and } 3 \\
& J_{i} \quad \text { a set of integers which indicate the neighborhood of } \\
& i \text {-th grid block } \\
& k \quad \text { permeability, darcies }\left[\mathrm{m}^{2}\right] \\
& k_{i} \quad \text { permeability values at the } i \text {-th simulator grid, darcies }\left[m^{2}\right] \text {, } \\
& i=1, \ldots, N \\
& \ell_{x}, l_{y} \quad \text { indices for bi-cubic spline approximation grid, } \ell_{x}=1, \ldots \text {, } \\
& N_{x s} ; l_{y}=1, \ldots, N_{y s} \\
& \mathrm{~N} \quad \mathrm{~N}_{x} \mathrm{~N}_{y} \text {, total number of simulator grid blocks } \\
& N_{\text {obs }} \quad \text { number of observation locations } \\
& \mathrm{N}_{\mathrm{W}} \quad \text { number of wells } \\
& \mathrm{N}_{\mathrm{t}} \quad \text { number of observation times } \\
& \mathrm{N}_{x}, \mathrm{~N}_{y} \quad \text { number of simulator grid blocks along } x \text { - and } y \text {-directions } \\
& \mathrm{N}_{\mathrm{xs}}, \mathrm{N}_{\mathrm{ys}} \quad \text { number of nodes along } \mathrm{x} \text { - and } \mathrm{y} \text {-direction of spline grid } \\
& \mathrm{N}_{\mathrm{N}} \quad \text { set of integers from } 1 \text { to } \mathrm{N} \\
& N_{x}, N_{y} \quad \text { set of integers from } 1 \text { to } N_{x} \text { and } N_{y}
\end{aligned}
$$




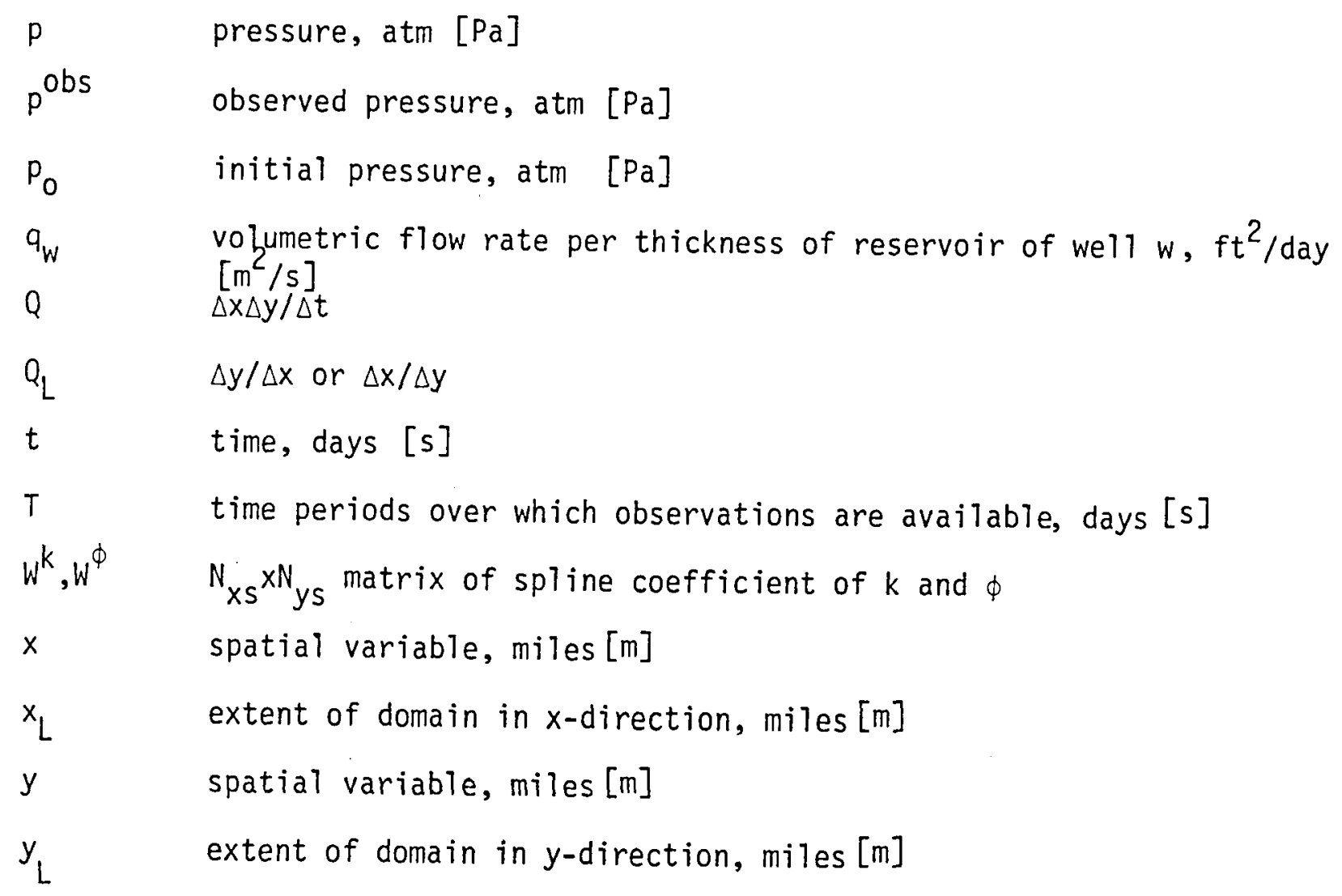

\section{Greek Letters}
$\alpha \quad$ unknown parameter to be estimated ( $\alpha=k$ or $\phi)$
$B_{k} \quad$ regularization parameter for the estimation of $k$, $\operatorname{atm}^{2} /$ darcies $^{2}\left[\mathrm{~Pa}^{2} / \mathrm{m}^{2}\right]$
$\beta_{\phi} \quad$ regularization parameter for the estimation of $\phi, \operatorname{atm}^{2}\left[\mathrm{~Pa}^{2}\right]$
$\Delta t \quad$ time interval of observation, days [s]
$\Delta x, \Delta y \quad$ simulator grid spacings, mile [m]
$\Delta \mathrm{x}_{\mathrm{s}}, \Delta \mathrm{y}_{\mathrm{s}} \quad$ spline grid spacings, mile [m]
$\delta(\cdot) \quad$ Dirac delta function
$\delta_{i, j} \quad$ Kronecker delta
$\zeta_{m+1} \quad$ weighting factor of Sobolev norm $\|\cdot\|_{H^{3}(\Omega)}, m=0,1,2$, and 3 $n \quad y / \Delta y$
$\mu \quad$ viscosity, centipoise [Pa.s]




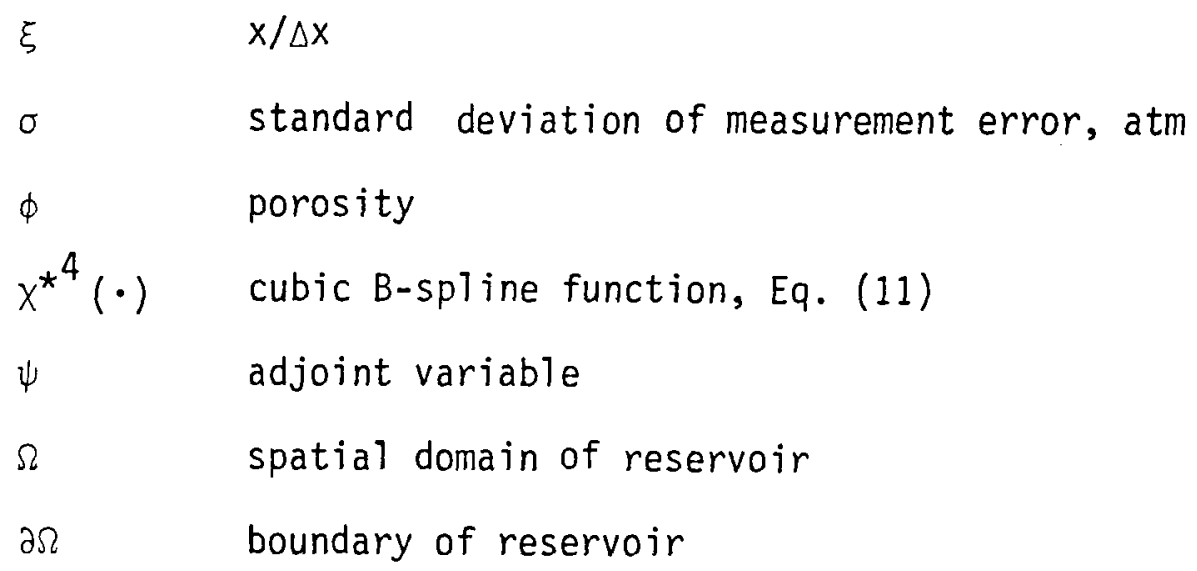

SI Metric Conversion Factors

$\begin{array}{lll}\text { atm } & \times 1.01325^{*} & E+05=P a \\ \text { atm }^{2} / \text { darcies }^{2} & \times 1.054 & E+34=\mathrm{Pa}^{2} / \mathrm{m}^{4} \\ \text { centipoise } & \times 1^{*} & \mathrm{E}-03=\mathrm{Pa} . \mathrm{s} \\ \text { darcies } & \times 9.869 & \mathrm{E}-13=\mathrm{m}^{2} \\ \text { days } & \times 1.157 & \mathrm{E}-05=\mathrm{s} \\ \mathrm{ft}^{2} / \text { day } & \times 1.075 & \mathrm{E}-0.6=\mathrm{m}^{2} / \mathrm{s} \\ \mathrm{miles} & \times 1.609344 * & \mathrm{E}+03=\mathrm{m}\end{array}$

*Conversion factor is exact. 


\section{REFERENCES}

1. Shah, P.C., Gavalas, G.R., and Seinfeld, J.H., "Error Analysis in History Matching: The Optimum Level of Parametrization," Soc. Pet. Eng. J. (June 1978) pp. 219-228.

2. Yakowitz, S. and Duckstein, L., "Instability in Aquifer Identification: Theory and Case Studies," Water Resources Research (Volume 16, 198) pp. 1054-1064.

3. Kravaris, C. and Seinfeld, J.H., "Identification of Parameters in Distributed Parameter Systems by Regularization," SIAM J. Control and Optimization, (Vol. 23, No. 2) pp. 217-241.

4. Kravaris, C. and Seinfeld, J.H. "Identification of Spatially-Varying Parameters in Distributed Parameter Systems by Discrete Regularization," J. Mathematical Analysis and Appl., in press

5. Banks, H.T., "Distributed System Optimal Control and Parameter Estimation: Computational Techniques Using Spline Approximations," Proceedings 3rd IFAC Symposium on Control of Distributed Parameter Systems, Toulouse, France, June 29 - July 2, 1982.

6. Banks, H.T. and Crowley, J.M. "Parameter Identification in Continuum Models," American Control Conference, San Francisco (June 1983).

7. Banks, H.T. and Murphy, K.A., "Estimation of Coefficients and Boundary Parameters in Hyperbolic Systems," Division of Applied Mathematics, Brown University, Report LCDS No. 84-5 (Feb. 1984).

8. Banks, H.T. and Lamm, P.D., "Estimation of Variable Coefficients in Parabolic Distributed Systems," I.E.E.E. Trans. Auto. Control, (Vol. 30, No. 4) pp 386-398.

9. Nazareth, L., "A Conjugate Direction Algorithm Without Line Searchers," J. Optimization Theory and Application (Nov. 1977) pp. 373-387.

10. Kravaris, C. and Seinfeld, J.H., "Identifiability of Spatially-Varying Conductivity from Point observation as an Inverse Sturm-Liouville Problem," SIAM J. Control and Optimization, (Vol. 24, No. 3) pp. XXXXXX.

11. Carter, R.D., Kemp, L.F. and Williams, D., "Performance Matching With Constraints," Soc. Pet. Eng. J. (April 1974) pp. 187-196.

12. Chen, W.H., Gavalas, G.R., Seinfeld, J.H., and Wassermann, M.L., "A New Algorithm for Automatic History Matching," Soc. Pet. Eng. J. (Dec. 1974) pp. 593-608.

13. Chavent, G., Dupuy, M., and Lemonnier, P., "History Matching by Use of Optimal Control Theory," Soc. Pet. Eng. J. (Feb. 1975) pp. 74-86.

14. Wassermann, M.L., Emanuel, A.S., and Seinfeld, J.H., "Practical Applications of Optimal Control Theory to History Matching Multiphase Simulator Models," Soc. Pet. Eng. J. (Aug. 1975) pp. 347-355. 
15. Dogru, A.H. and Seinfeld, J.H., "Comparison of Sensitivity Coefficient Calculation Methods in Automatic History Matching," Soc. Pet. Eng. J. (0ct. 1981) pp. 551-557.

16. Lawson, C.L. and Hansen, R.J., Solving Least Square Problems, PrenticeHall, Englewood Cliffs, N.J. (1974).

17. Scales, L.E., Introduction to Non-Linear Optimization, Springer-Verlag New York Inc., New York, NY (1985).

18. Tikhonov, A.N., and Arsenin, V.Y., Solutions of Ill-posed Problems, V. H. Winston \& Sons, Washington, D.C., (1977). 
Table 1. Specifications of Reservoir for History Matching Example

$\begin{array}{lll} & \text { Oilfield units } & \text { SI units } \\ \text { Dimension of reservoir } & 12.4 \times 18.6(\mathrm{mi}) & 20,000 \times 30,000(\mathrm{~m}) \\ \text { Compressibility of system } & 1.2 \times 10^{-5}\left(\mathrm{~atm}^{-1}\right) & 1.2 \times 10^{-10}\left(\mathrm{~Pa}^{-1}\right) \\ \text { Viscosity of fluid } & 2.0(\mathrm{cp}) & 2.0 \times 10^{-3}(\mathrm{~Pa} . \mathrm{s}) \\ \text { Number of production wells } & 1 & 1 \\ \text { Production rate } & 500\left(\mathrm{ft}^{2} / \text { day) }\right. & 5.376 \times 10^{-4}\left(\mathrm{~m}^{2} / \mathrm{s}\right) \\ \text { Initial pressure } & 150(\mathrm{~atm}) & 1.52 \times 10^{7}(\mathrm{~Pa}) \\ \text { Pressure grid } & 10 \times 15 & 10 \times 15 \\ \text { Number of observation wells } & 6 & 6 \\ \text { Number of well pressure data per each well } & 35 & 35 \\ \text { Time interval of well pressure data } & 10(\text { days }) & 8.64 \times 10^{6}(\mathrm{~s}) \\ \text { Total number of data points } & 210 & 210\end{array}$


Table 2. True Values of Porosity and Permeability, the Uniform Values of $\phi$ and $k$ that Minimize $J_{L S}$, and the Corresponding starting values of $J_{L S}, J_{S T}$, and $J_{S M}$

\begin{tabular}{|c|c|c|c|c|}
\hline $\begin{array}{l}\text { Parameter to be } \\
\text { estimated }\end{array}$ & \multicolumn{2}{|c|}{$\phi$} & \multicolumn{2}{|c|}{$k^{(1)}$} \\
\hline True VaTue & \multicolumn{2}{|c|}{$\begin{array}{l}0.2-0.05 \sin \left(\pi x / x_{L}\right) \\
\cdot \sin \left(2 \pi y / y_{L}\right)\end{array}$} & \multicolumn{2}{|c|}{$\begin{array}{l}0.3-0.1 \sin \left(\pi x / x_{L}\right) \\
\cdot \sin \left(2 \pi y / y_{L}\right)\end{array}$} \\
\hline Initial guess & \multicolumn{2}{|c|}{0.25 and 0.15} & \multicolumn{2}{|c|}{0.25 and 0.35} \\
\hline $\begin{array}{l}\bar{\alpha} \text { that minimizes } \\
J_{L S}\end{array}$ & \multicolumn{2}{|c|}{0.184} & \multicolumn{2}{|c|}{0.241} \\
\hline $\mathrm{J}_{\mathrm{LS}}$ & \multicolumn{2}{|c|}{44.6} & \multicolumn{2}{|c|}{47.0} \\
\hline $\mathrm{J}_{S T}$ & \multicolumn{2}{|c|}{5.1} & \multicolumn{2}{|c|}{8.7} \\
\hline \multirow[t]{5}{*}{$\mathrm{J}_{S M}$} & $\phi=0^{(2)}$ & 44.6 & $\beta_{k}=0^{(3)}$ & 41.0 \\
\hline & 0.01 & 44.7 & 0.01 & 41.1 \\
\hline & 0.1 & 45.1 & 0.1 & 41.9 \\
\hline & 1 & 49.7 & 1 & 49.7 \\
\hline & 10 & 95.6 & 10 & 128.0 \\
\hline
\end{tabular}
(1) units are darcies
(2) units are $\mathrm{atm}^{2}$
(3) units are $a t m^{2} /$ darcies $^{2}$ 
Table 3. Estimation of $\phi$

(a) Final values of performance indices for $\sigma=0.3 \mathrm{~atm}$ and $N_{x s} \times N_{y s}=7 \times 9$ as a function of $\beta_{\phi}\left(a t m^{2}\right)$

\begin{tabular}{llllllll}
\hline$B_{\phi}$ & $J_{S M}$ & $J_{L S}$ & $J_{S T}{ }^{a}$ & $J_{S T}^{(1)}$ & $J_{S T}^{(2)} \times 10^{2}$ & $J_{S T}^{(3)} \times 10^{3}$ & $J_{S T}^{(4)} \times 10^{3}$ \\
\hline 0 & 20.67 & 20.67 & 15.55 & 6.47 & 5.30 & 30.24 & 193.63 \\
0.01 & 20.86 & 20.76 & 9.47 & 6.47 & 2.67 & 10.96 & 61.64 \\
0.1 & 21.60 & 20.85 & 7.48 & 6.44 & 1.76 & 5.11 & 18.75 \\
1 & 27.61 & 27.16 & 6.45 & 6.28 & 0.75 & 1.47 & 1.79 \\
10 & 81.69 & 24.09 & 5.76 & 5.74 & 0.20 & 0.12 & 0.09 \\
true & & & 7.09 & 6.17 & 2.63 & 7.48 & 1.88
\end{tabular}

${ }^{a} \zeta_{1}=1, \zeta_{2}=h^{2}, \zeta_{3}=h^{4}, \zeta_{4}=h^{6}$, with $h=2.5$

(b) Final values of performance indices for $\sigma=0.3 \mathrm{~atm}$ and $\beta_{\phi}=1 \mathrm{~atm}^{2}$ as a function of $\mathrm{N}_{x s} \times N_{y s}$

\begin{tabular}{lllllllll}
\hline$N_{x S} \times N_{y S}$ & $h$ & $J_{S M}$ & $J_{L S}$ & $J_{S T}{ }^{a}$ & $J_{S T}^{(1)}$ & $J_{S T}^{(2)} \times 10^{2}$ & $J_{S T}^{(3)} \times 10^{3}$ & $J_{S T}^{(4)} \times 10^{3}$ \\
\hline $5 \times 6$ & 5 & 27.86 & 21.38 & 6.47 & 6.29 & 0.50 & 0.04 & 0.002 \\
$7 \times 9$ & 2.5 & 27.61 & 21.16 & 6.45 & 6.28 & 0.75 & 1.47 & 1.79 \\
$12 \times 17$ & 1.09 & 27.17 & 20.78 & 6.40 & 6.31 & 3.11 & 18.38 & 14.78
\end{tabular}

${ }^{a} \xi_{1}=1, \xi_{2}=h^{2}, \xi_{3}=h^{4}, \xi_{4}=h^{6}$ 
Table 4. Estimation of $k$

(a) Final values of performance indices for $\sigma=0.32 \mathrm{~atm}$ and $N_{x s} \times N_{y s}=7 \times 9$ as a function of $\beta_{k}\left(a^{2} / \text { darcies }^{2}\right)^{5}$

\begin{tabular}{lcrrrrrr}
\hline$\beta_{k}$ & $J_{S M}$ & $J_{L S}$ & $J_{S T}{ }^{a}$ & $J_{S T}^{(1)}$ & $J_{S T}^{(2)} \times 10^{2} J_{S T}^{(3)} \times 10^{3} J_{S T}^{(4)} \times 10^{3}$ \\
\hline 0 & 21.26 & 21.26 & 20.46 & 11.68 & 7.81 & 36.75 & 28.05 \\
0.01 & 21.59 & 21.44 & 14.86 & 11.36 & 5.56 & 18.14 & 9.99 \\
0.1 & 22.82 & 21.49 & 13.36 & 11.38 & 4.79 & 12.33 & 4.84 \\
1 & 33.42 & 22.18 & 11.24 & 10.54 & 3.83 & 5.36 & 1.04 \\
10 & 114.7 & 35.70 & 7.90 & 7.39 & 4.98 & 2.86 & 0.33 \\
true & & & 17.54 & 13.88 & 10.50 & 29.97 & 7.50 \\
\hline
\end{tabular}

${ }^{a} \zeta_{1}=1, \zeta_{2}=h^{2}, \zeta_{3}=h^{4}, \zeta_{4}=h^{6}$ with $h=2.5$

(b) Final values of performance indices for $\sigma=0.3 \mathrm{~atm}$ and $\beta_{k}=1 \mathrm{~atm}^{2} /$ darcies $^{2}$ as a function of $\mathrm{N}_{\mathrm{xs}} \mathrm{xN}_{\mathrm{ys}}$

$N_{x S} x N_{y S} \quad h \quad J_{S M} \quad J_{L S} \quad J_{S T}{ }^{a} \quad J_{S T}^{(1)} \quad J_{S T}^{(2)} \times 10^{2} J_{S T}^{(3)} \times 10^{3} \quad J_{S T}^{(4)} \times 10^{3}$

$\begin{array}{lllllllll}5 \times 6 & 5 & 35.70 & 23.48 & 12.22 & 11.30 & 1.30 & 0.39 & 0.02\end{array}$

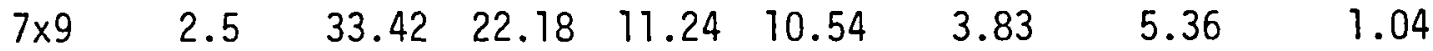

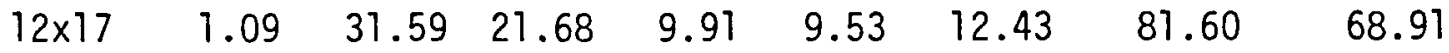

${ }^{a} \zeta_{1}=1, \zeta_{2}=h^{2}, \xi_{3}=h^{4}, \xi_{4}=h^{6}$.

(c) Final values of performance indices for $\sigma=0.3 \mathrm{~atm}, \beta_{k}=$ $1 \mathrm{~atm}^{2} /$ darcies $^{2}$, and $\mathrm{N}_{\mathrm{xs}} \times \mathrm{N}_{\mathrm{ys}}=7 \times 9$ with different numbekr of observation wells

\begin{tabular}{cccccccc}
\hline $\begin{array}{l}\text { \# of } \\
\text { wel1s }\end{array}$ & $J_{S M}$ & $J_{L S}$ & $J_{S T}{ }^{a}$ & $J_{S T}^{(1)}$ & $J_{S T}^{(2)} \times 10^{2}$ & $J_{S T}^{(3)} \times 10^{3}$ & $J_{S T}^{(4)} \times 10^{3}$ \\
\hline 6 & 33.42 & 22.18 & 11.24 & 10.54 & 3.83 & 5.36 & 1.04 \\
18 & 76.96 & 63.33 & 13.62 & 11.96 & 5.00 & 11.28 & 3.74 \\
\hline
\end{tabular}

$a_{\zeta_{1}}=1, \zeta_{2}=h^{2}, \zeta_{3}=h^{4}, \zeta_{4}=h^{6}$ with $h=2.5$.

${ }^{\mathrm{b}}$ To convert from $\mathrm{atm}^{2} /$ darcies $^{2}$ to SI units see Notation. 
Table 5. Values of $\left\|\beta_{\alpha} \frac{d W^{\alpha}}{d \beta_{\alpha}}\right\|_{2}$

(a) Estimation of $\phi$

\begin{tabular}{lll}
\hline$\beta_{\phi}$ & $\left\|\beta_{\phi} \mathrm{dW}^{\phi} / \mathrm{d} \beta_{\phi}\right\|_{2}$ \\
\hline $0.01,0.1$ & 0.167 \\
$0.1,1$ & 0.148 \\
$1, \quad 10$ & 0.169 \\
\hline
\end{tabular}

(b) Estimation of $\mathrm{k}$

\begin{tabular}{ll}
\hline \multicolumn{1}{c}{$\beta_{k}$} & $\left\|\beta_{k} d W^{k} / d \beta_{k}\right\|_{2}^{a}$ \\
\hline $0.01,0.1$ & 0.211 \\
$0.1,1$ & 0.229 \\
1,10 & 0.456 \\
\hline
\end{tabular}

anits are darcies. To convert to SI units see Notation. 
FIGURE CAPTIONS

Figure 1. Pressure and spline grid system, pressure grid: 10x15;

$$
\text { spline grid: } \quad 5 \times 6 ; \quad 7 \times 9 ; \quad 12 \times 77
$$

$(0=$ Observation wel1; $P=$ production well)

Figure 2. True $\phi(x, y)$ surface

$$
\phi(x, y)=0.2-0.05 \sin \left(\pi x / x_{L}\right) \sin \left(2 \pi y / y_{L}\right)
$$

Figure 3. True $k(x, y)$ surface

$$
k(x, y)=0.3-0.1 \sin \left(\pi x / x_{L}\right) \sin \left(2 \pi y / y_{L}\right) \text { darcies }
$$

Figure 4. Simulated pressure data vs. time for $\sigma=0.3 \mathrm{~atm}$

$$
\begin{aligned}
& \text { 1. }(3.1,3.1)^{\mathrm{a}} \\
& \text { 2. }(9.3,3.1) \\
& \text { 3. }(3.1,9.3) \\
& \text { 4. }(9.3,9.3) \\
& \text { 6. }(3.1,15.5) \\
& \text { 6. }(9.3,15.5) \\
& \text { aunits are miles }
\end{aligned}
$$

Figure 5. Estimated $\phi$ surface for $\sigma=0.3 \mathrm{~atm}, \mathrm{~N}_{x s} \times N_{y s}=7 \times 9$, and from ton down:

$$
\begin{gathered}
\beta_{\phi}=0 \mathrm{~atm}^{2} \\
0.01 \\
0.1 \\
1 \\
10
\end{gathered}
$$

Figure 6. Cross-sectional plot of $k\left(x_{L} / 2, y\right)$ vs. $y$ for $\sigma=0.3 \mathrm{~atm}$ $\mathrm{N}_{x s} \times N_{y s}=7 \times 9$ and

$$
\begin{array}{ll}
\text { 1. } & \beta_{\mathrm{k}}= \\
\text { 2. } & 0.01 \\
\text { 3. } & 0.1 \\
\text { 4. } & 1 \\
\text { 5. } & 10 \\
\text { 6. } & \text { true values } \\
\text { 7. } & \text { initial guess }
\end{array}
$$

Figure 7. Cross-seçtional plot of $\phi\left(x_{L} / 2 y\right)$ vs. $y$ for $\sigma=0.3 \mathrm{~atm}$, $B_{\phi}=1 \mathrm{~atm}^{2}$, and
1. $N_{x s} \times N_{y s}=5 \times 6$
2. $7 \times 9$
3. $12 \times 17$
4. true values
5. initial guess

Figure 8. Estimated $k$ surface for $\sigma=0.3 \mathrm{~atm}, B_{k}=1 \mathrm{~atm}^{2} /$ darcies $^{2}$ and

$$
\begin{aligned}
\text { from top down: } & 5 \times 6 \\
\mathrm{NSS}_{\mathrm{ys}}= & 5 \times 6 \\
& 7 \times 9 \\
& 12 \times 17
\end{aligned}
$$


Figure 9. Estimated and true $k$ surfaces for $\sigma=0.3 \mathrm{~atm}$, $\beta_{k}=1 \mathrm{~atm}^{2} /$ darcies $^{2}, N_{x s} \times N_{y s}=7 \times 9$, and from top down:

6 observation wells 18 observation wells true $k$ 

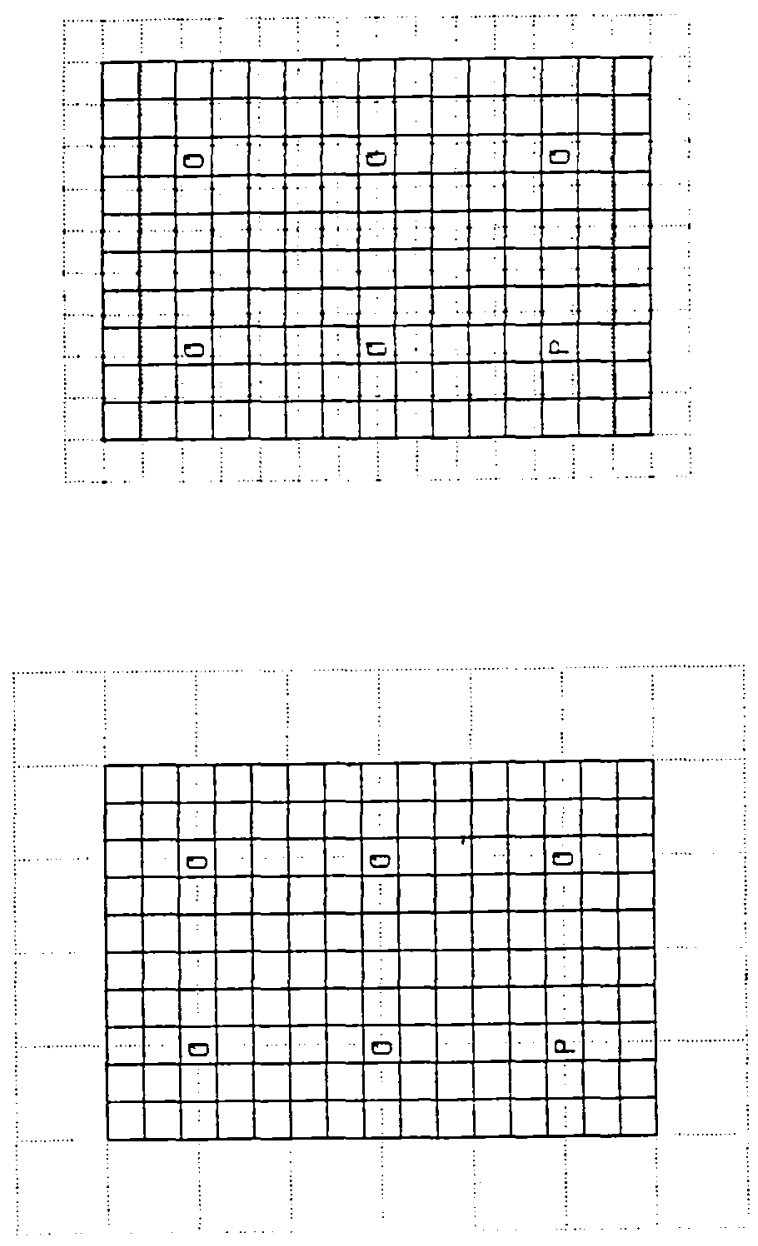

ص.

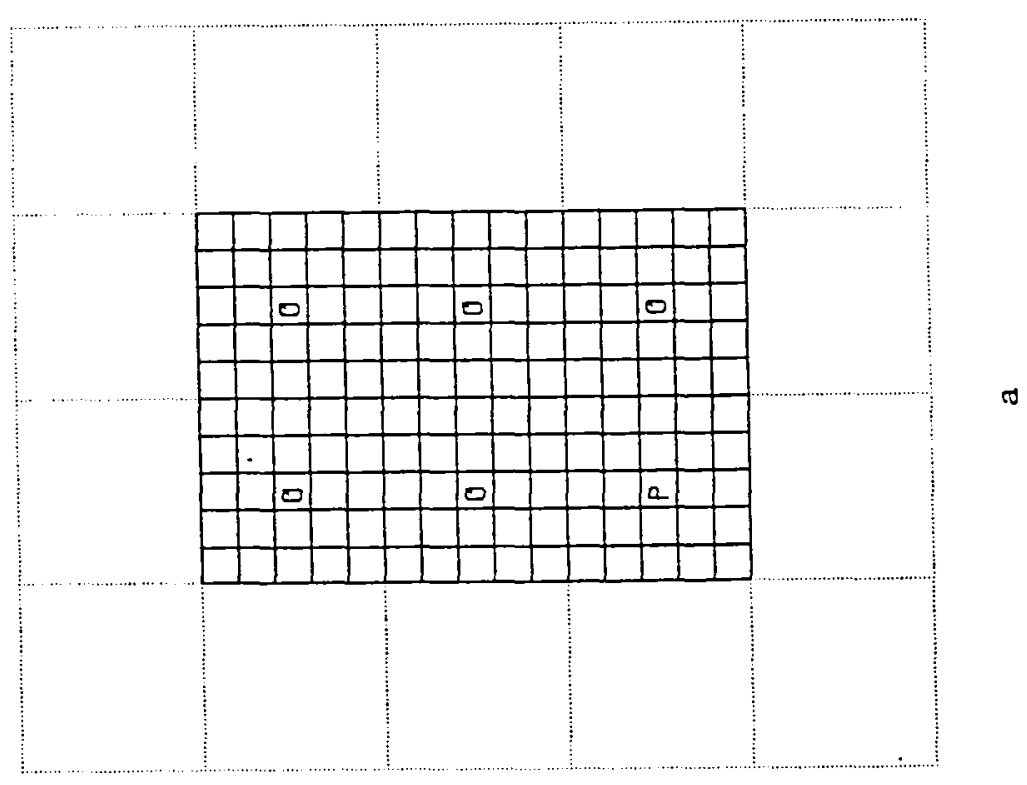

Figure 1 


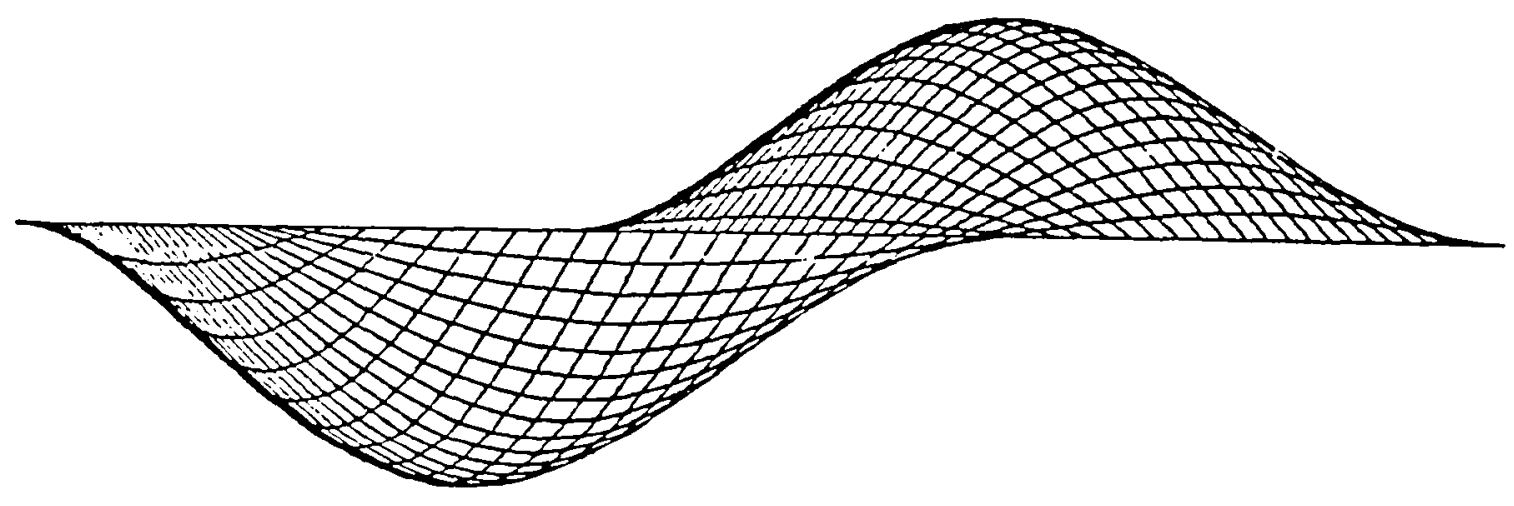

Figure 2 


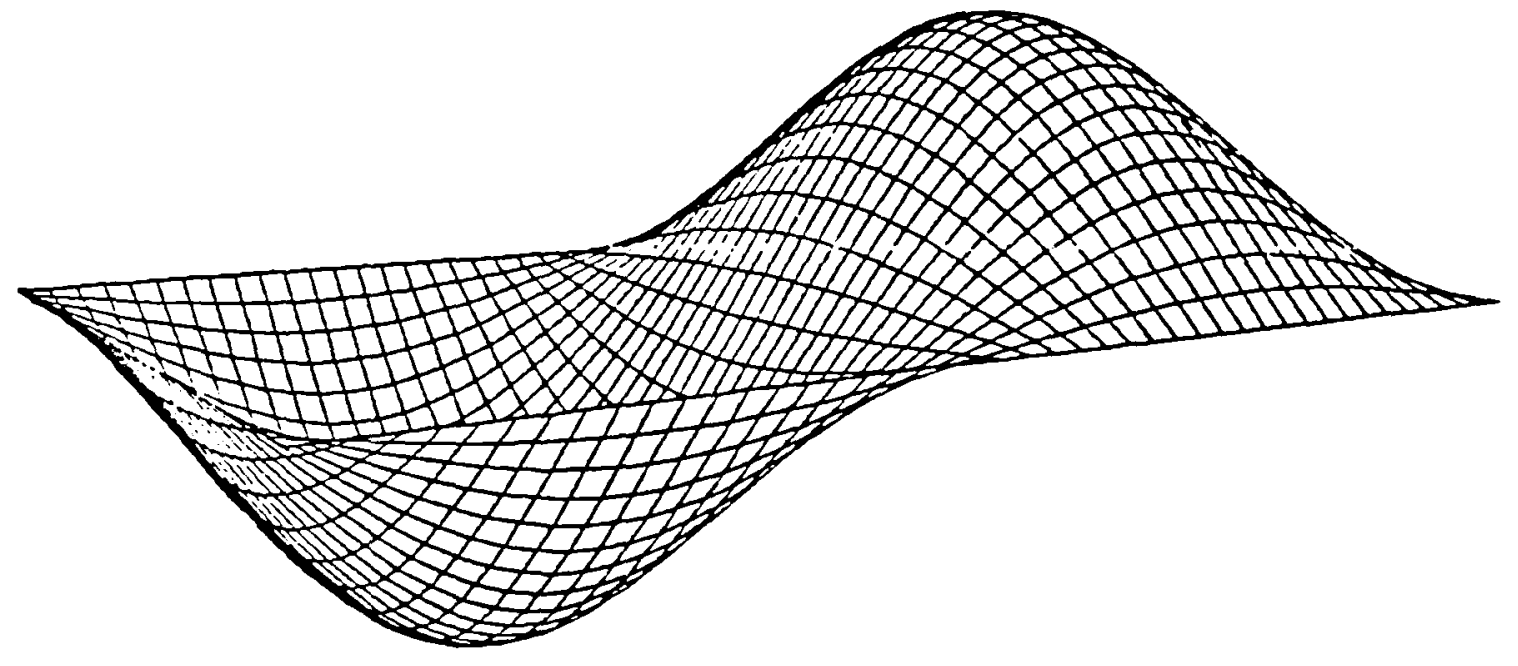

Figure 3 


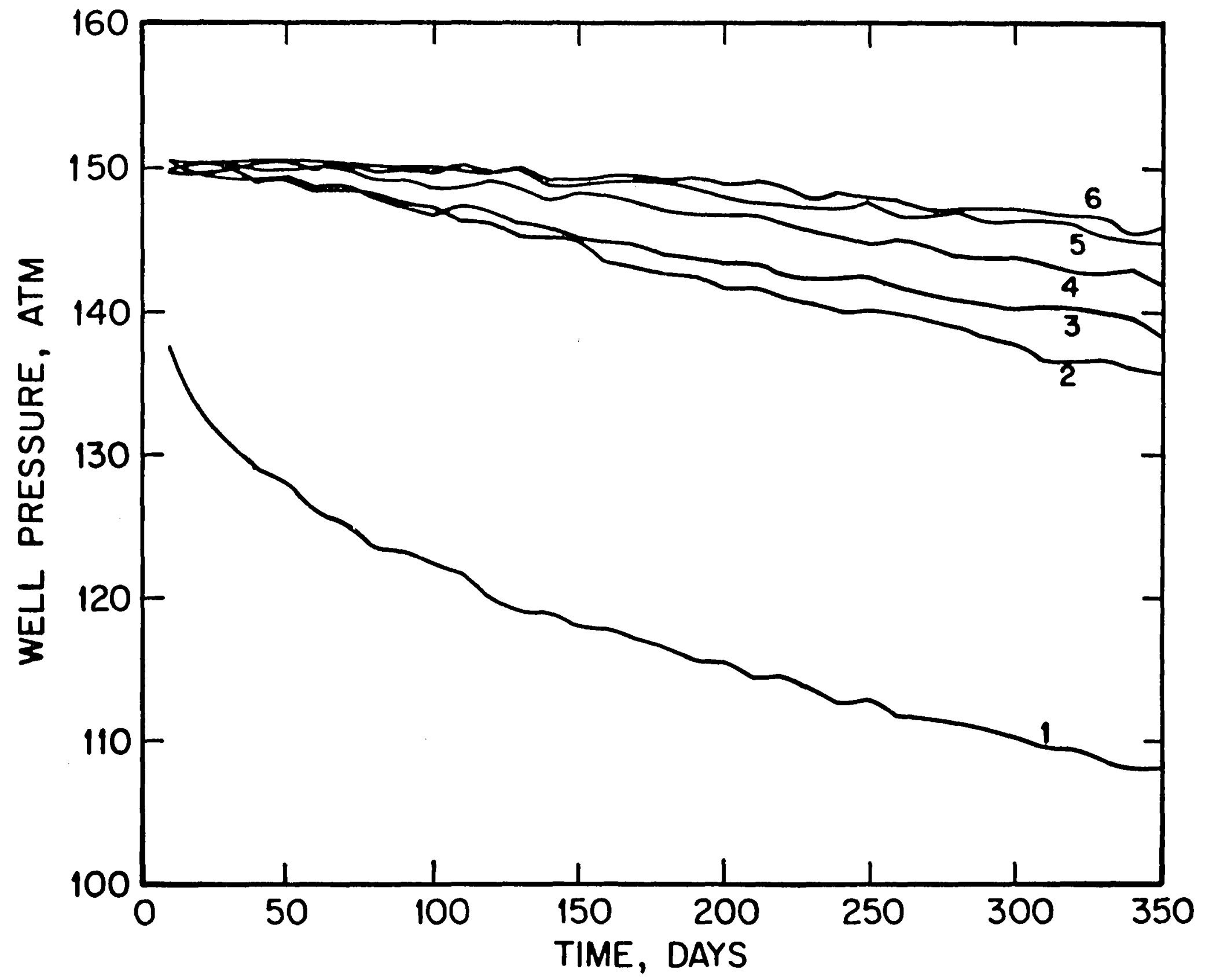



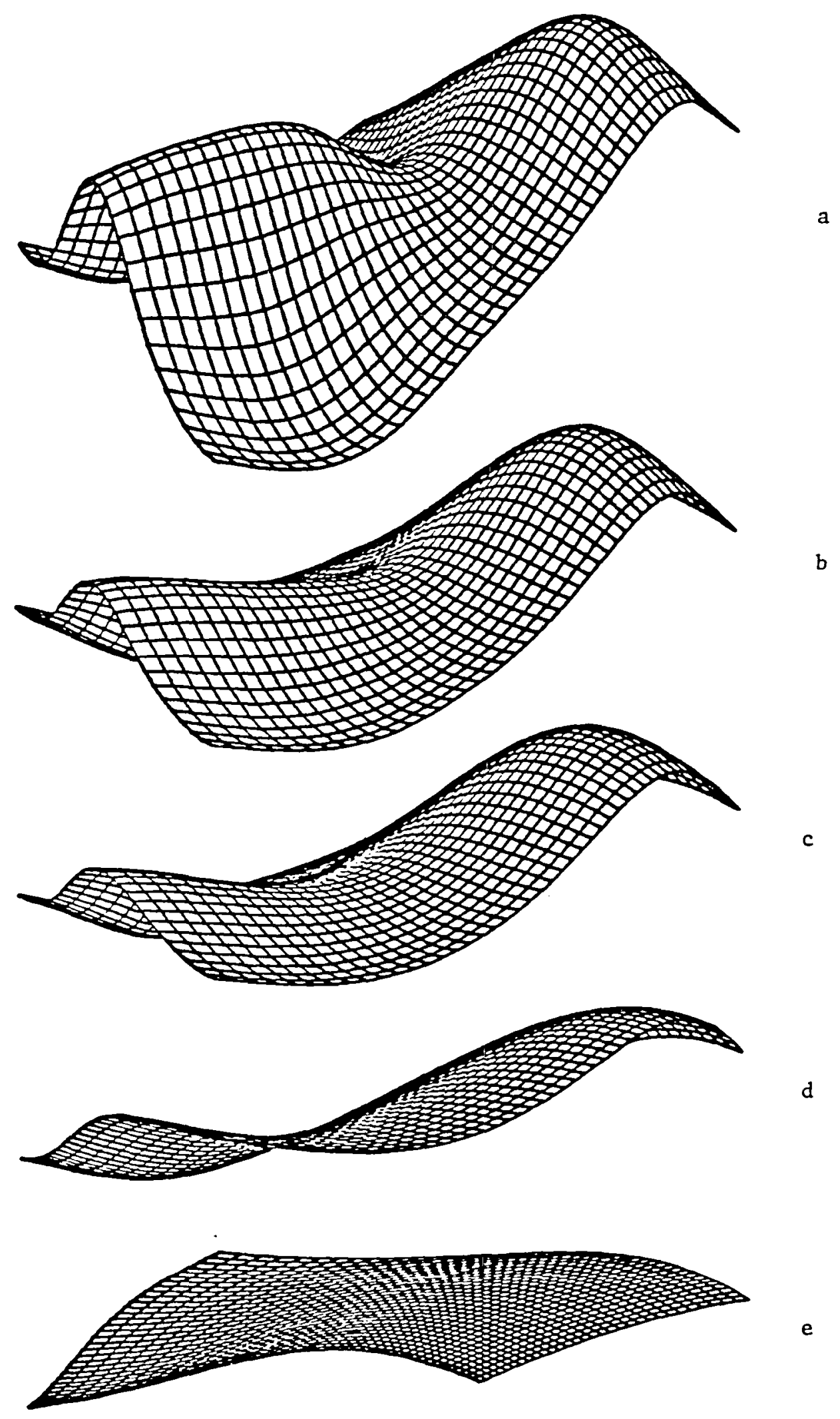

Figure 5 


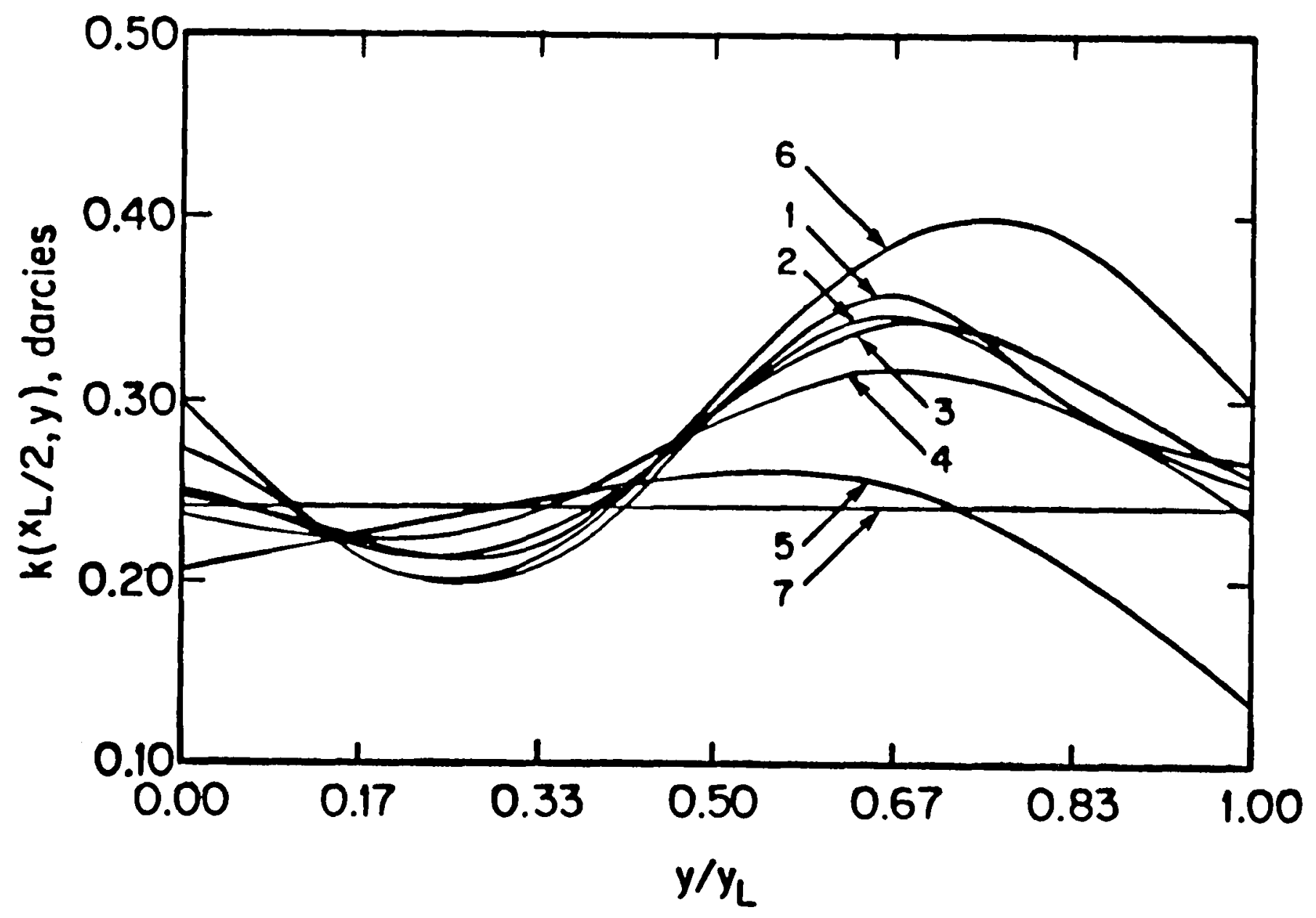

Figure 6 


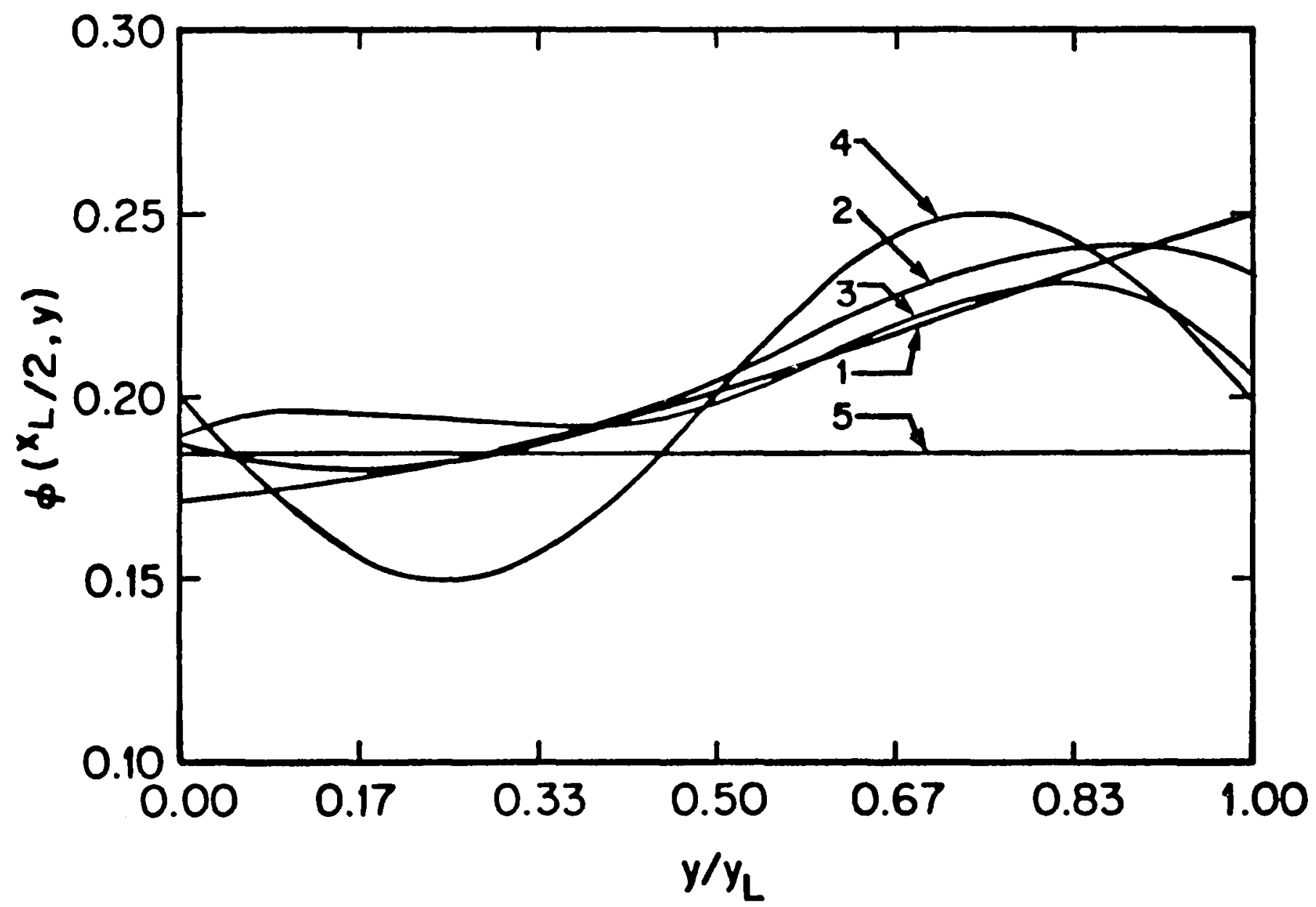

Figure 7 

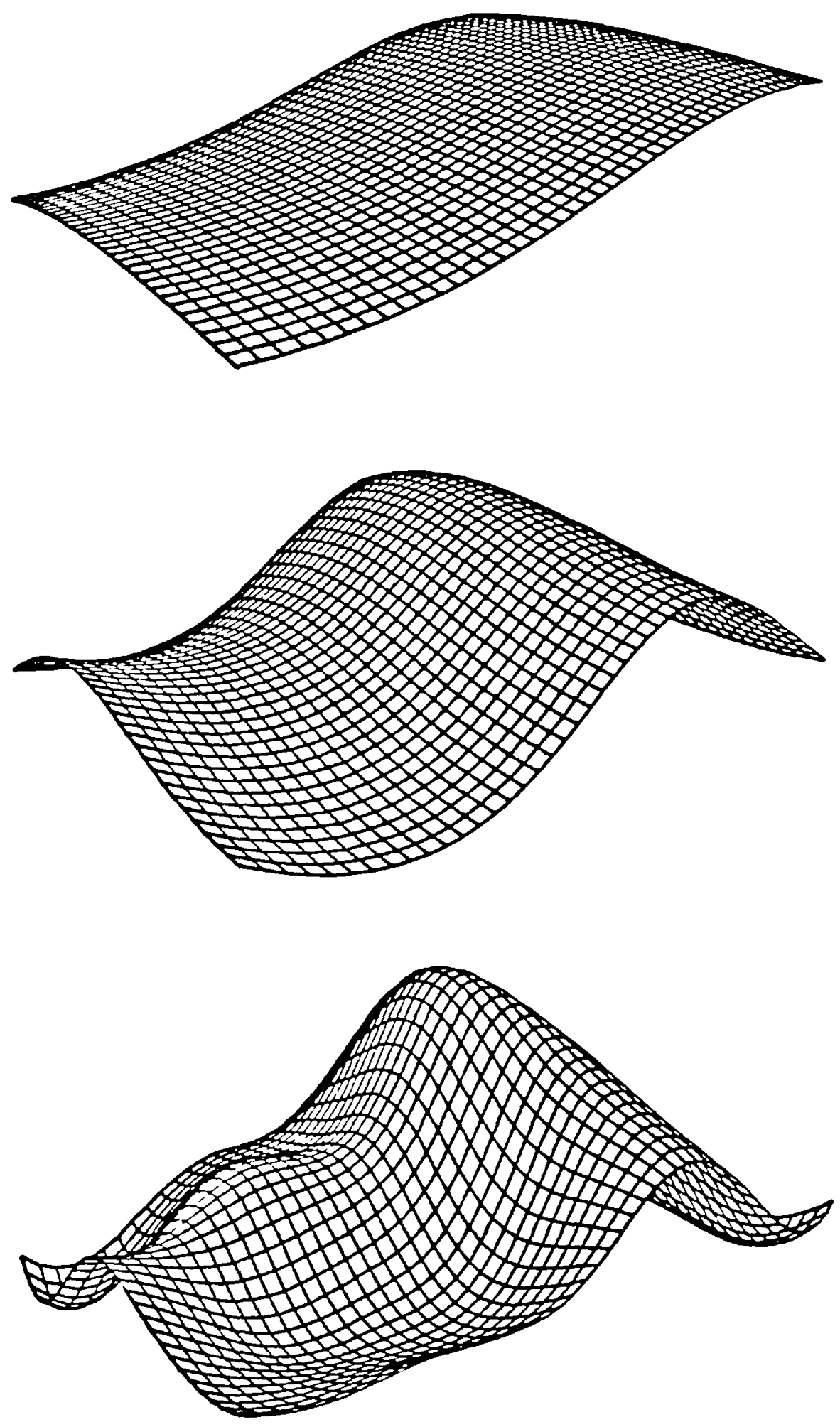

c

Figure 8 

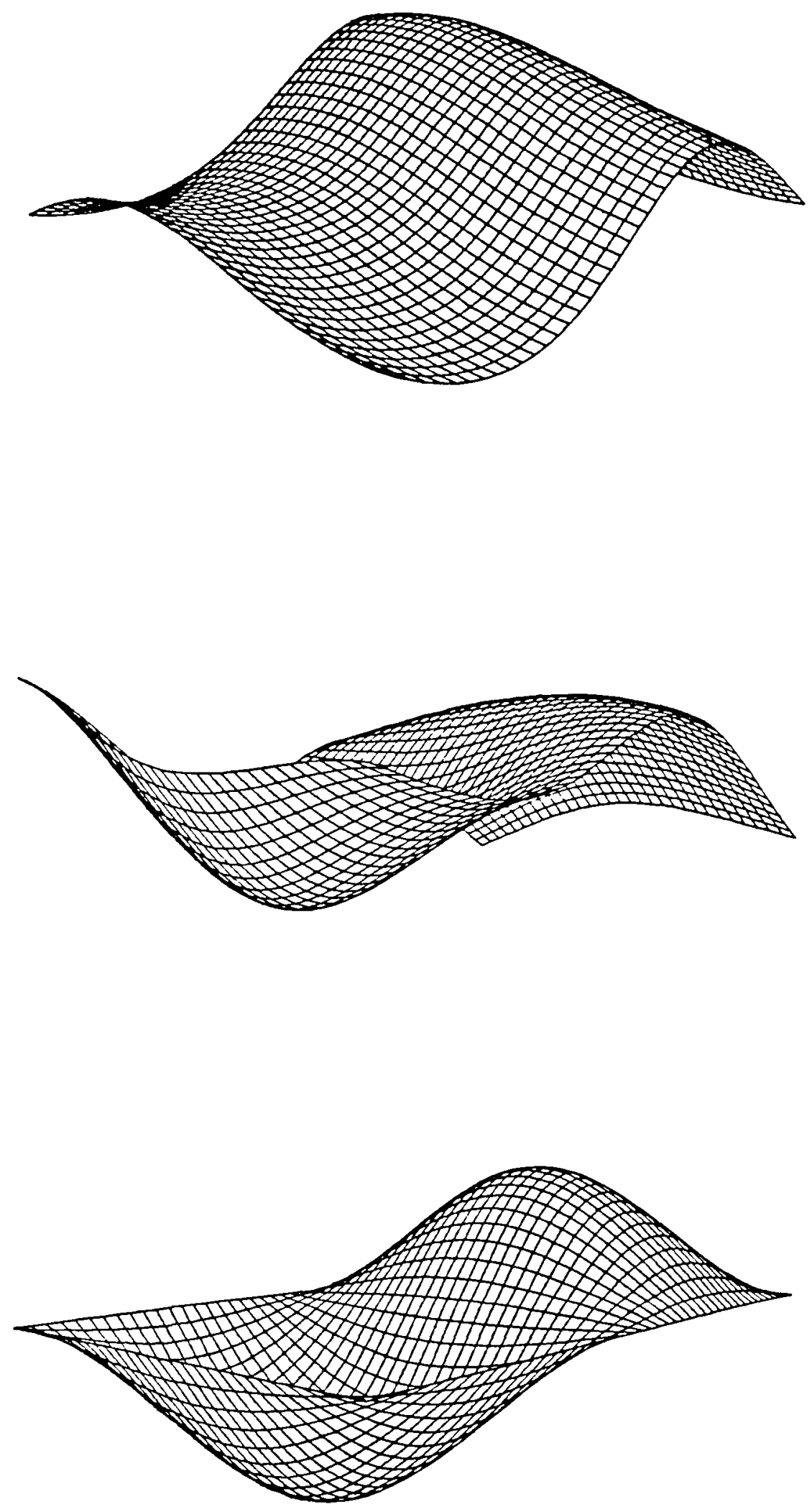

Figure 9 




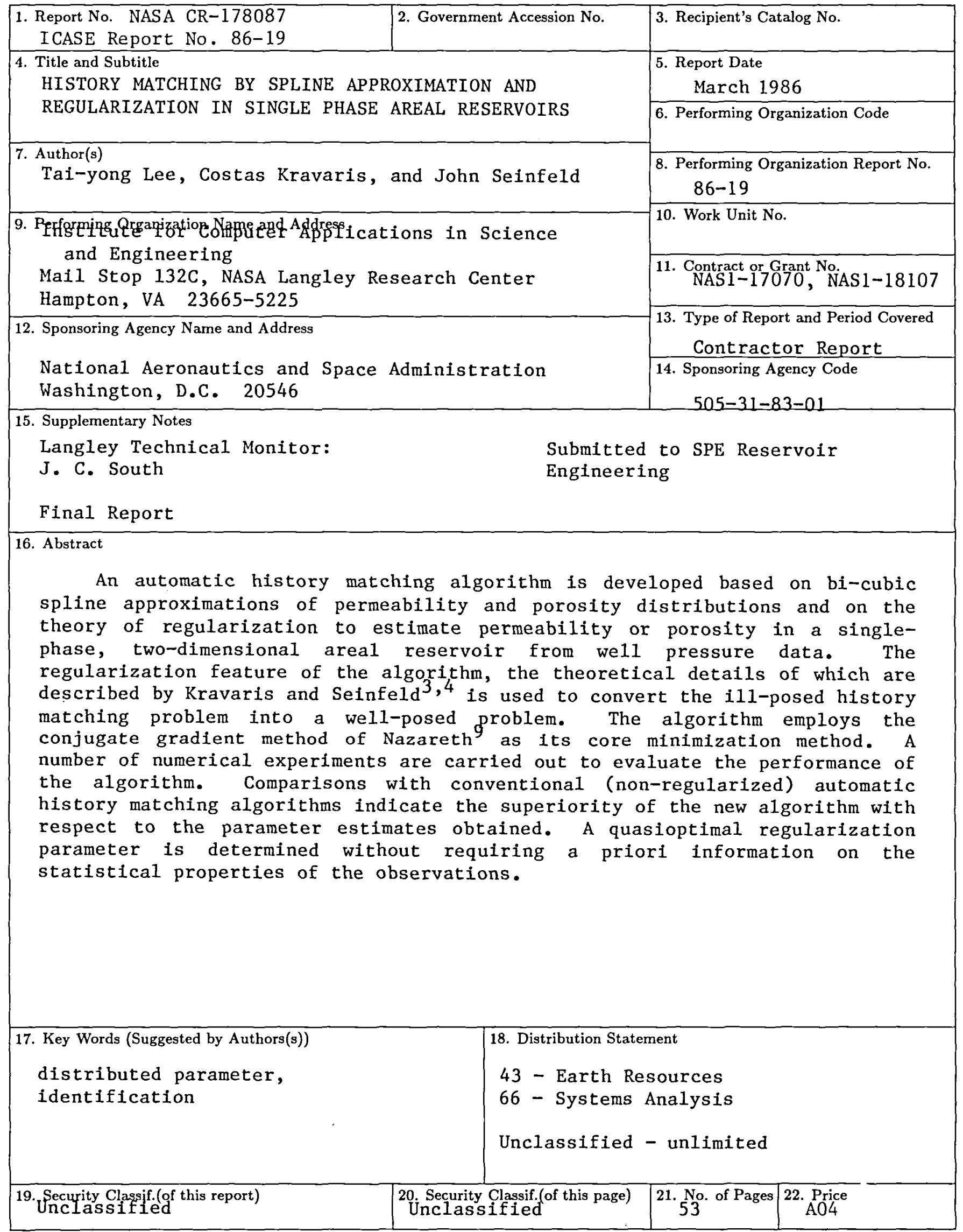



